\title{
Abstracts for the 38th Human Genetics Society of Australasia Annual Scientific Meeting Adelaide, South Australia
}

August 3-6, 2014

\section{Plenaries and Orals}

\section{Plenary 1 \\ WHAT'S THE FUSS ABOUT INCIDENTAL FINDINGS? OPPORTUNISTIC SCREENING AND INTERNATIONAL ATTITUDES}

\section{Anna Middleton}

Wellcome Trust Sanger Institute, Hinxton, Cambridge, UK

While whole genome/exome sequencing in a research setting may be used to explore the genetic basis of a phenotype it also offers the chance to opportunistically screen for additional results unrelated to the research project but relevant to the participants' future medical health (termed 'incidental findings', IFs).There is a wealth of medical and ethics literature supporting the feedback of IFs, yet there is limited empirical work offering a voice from both professional and public stakeholders directly affected by this. I will explore the current debate about sharing IFs in both a clinical and research setting and offer results from our web-based survey that has investigated the attitudes of 6,944 individuals from across 91 countries towards searching for and sharing incidental findings from genome research studies.

Eighty percent of participants believed that incidental findings from sequencing studies should be made available to research participants if they want them. Treatability and perceived usefulness of the data were important, with $98 \%$ personally interested in learning about life-threatening conditions that were preventable. However, only $31 \%$ of participants thought genomic researchers should actively search for incidental findings that were not relevant to their research study. Genetic health professionals held the most conservative views towards all aspects of data sharing. Ours is the largest dataset published to date on such attitudes. I will share an overview of how the debate about IFs has progressed in the United Kingdom. I will also offer information about the UK government initiative to sequence 100,000 genomes of patients in the National Health Service.

Plenary 2

COMMUNICATING ABOUT GENETICS: A FAMILY AFFAIR

Carma Bylund

Hamad Medical Corporation, Qatar

Plenary 3

DEVELOPMENT AND IMPLEMENTATION OF CLINICAL GENOMICS

John Mattick

Garvan Institute of Medical Research, Sydney, NSW, Australia
Plenary 4

FATTY ACID OXIDATION DEFECTS: PHENOTYPES, OUTCOME AND THE DIAGNOSTIC DILEMMA

Ute Spiekerkoetter

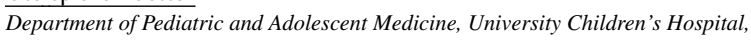
Freiburg, Germany

\section{Plenary 5}

NEWBORN SCREENING AND THE INTERFACE WITH TREATMENT

\section{Bridget Wilcken}

The Children's Hospital at Westmead and University of Sydney, Sydney, NSW, Australia

Newborn screening has been a success story for over 50 years. The early diagnosis of babies with serious, treatable disorders enables appropriate neonatal management, improved outcome, and may be life-saving. There are, however, some harms, particularly for patients with mild phenotypes who may not require any treatment. With the expansion of newborn screening to encompass more and rarer disorders this is becoming a major problem. Three examples have been chosen to illustrate mild phenotypes still not well-enough understood, what research is required and what is already ongoing to better define the need for treatment in babies with these mild forms of a disorder: the 'old' disorders of galactosaemia (Duarte/galactosemia compound heterozygote) and congenital hypothyroidism, and a newer inclusion in screening, very-long-chain acyl-CoA dehydrogenase deficiency (VLCADD). New treatments, especially mutation-specific therapies, mean that more disorders will be considered for inclusion in screening panels. New treatments also mean that long-term follow-up of all treated patients must be incorporated in newborn screening programs. The moves towards an agreed overarching policy for newborn screening in Australia will aid attainment of these aims locally.

\section{Plenary 6}

THE IDENTIFICATION OF NEW RNA GENES IN GWAS REGIONS ASSOCIATED WITH COMPLEX DISEASES

\section{John Mattick}

Garvin Institute of Medical Research, Sydney, NSW, Australia

\section{Plenary 7}

MORBIDITY INTELLECTUAL DISABILITY MAP OF HUMAN X-CHROMOSOME

\section{Jozef Gecz}

The University of Adelaide and SA Pathology, Women's and Children's Hospital, Adelaide, SA, Australia

Human X-chromosome represents about $5 \%$ of the human genome and $\sim 3 \%$ of the human exome. It contains 830 protein-coding 
genes (and 100s of pseudogenes and non-coding genes). Over the past 20 years a significant effort has been put into the identification of X-chromosome disease-causing DNA sequence variation, making the $\mathrm{X}$ one of the best studied human chromosomes. Of the protein-coding genes $\sim 110(13 \%)$ genes have been implicated (published and our unpublished data) in various forms of syndromic and non-sydromic intellectual disability. In addition to this multiple regions of the $\mathrm{X}$-chromosome have been involved in intellectual disability through structural variation, including copy number variation, translocation and inversion. I will present an overview of the $\mathrm{X}$-chromosome sequence variation in intellectual disability and how this summarized data is helping us to identify additional $\mathrm{X}$ chromosome regions likely to be implicated (or not) in intellectual disability as well as redefining the role of known XLID loci. I will also discuss some of our most recent XLID gene discoveries. In synthesizing our and international efforts so far to identify the majority if not all intellectual disability (and clinically relevant in general) loci on the X-chromosome I will point to the remaining challenges as well as to the genome-wide relevance of the

\section{Plenary 8 \\ MISSING VARIANTS IN HIGH THROUGHPUT SEQUENCING: METHODS FOR THE DETECTION OF DISEASE CAUSING VARIANTS}

Melanie Bahlo

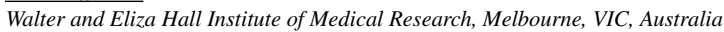

Next-generation sequencing (NGS) has led to an unprecedented increase in the discovery of disease causing variants. However, many family studies with strong linkage peaks remain unsolved despite repeated rounds of NGS. We describe some strategies with NGS data analysis that could lead to the discovery of a variant by incorporating familial and population data and extending the NGS analysis pipeline to look for more unusual causes of disease. We describe examples where the use of such information led to the identification of causal variants where standard filtering methods would not have succeeded or gotten to the answer in a timely manner.

\section{Plenary 9 \\ RESULTS FROM WHOLE GENOME AND EXOME SEQUENCING IN ID PATIENTS: A CLINICAL PERSPECTIVE}

\section{Bregje van Bon \\ Department of Human Genetics, Radboud University Medical Center, Nijmegen, the Netherlands and South Australian Clinical Genetics Service, SA Pathology, Women's and Children's Hospital, Adelaide, SA, Australia}

Intellectual disability (ID) occurs in $2-3 \%$ of the general population and the cause remains unknown in about $50 \%$ of patients. Knowing the cause allows anticipation on associated health and behavior problems and is essential for counseling regarding recurrence risk and prognosis. The extensive genetic heterogeneity of ID requires a genome wide detection of all types of genetic variation. Microarray studies and more recently exome sequencing have demonstrated the importance of de novo copy number variations and single nucleotide variations.

The implementation of exome sequencing in our diagnostic setting in December 2011 has led to significant changes in our daily clinical genetic practice, including pre-test counseling issues, interpretation of test-results in regard to the patient's phenotype and the approach to unexpected results. In the past 2 years, over 1,000 clinical exomes have been performed, including 400 ID patientparents trios. Workflow, experiences and pitfalls from a clinician's perspective will be discussed. Furthermore, the introduction of next generation sequencing techniques has led to the identification of novel recurrent genes within our ID cohort. An overview of several recently delineated syndromes will be presented.
Finally, in a subset of 50 individuals with severe ID, in whom micro-array-based CNV analysis and whole exome sequencing were negative, we applied whole genome sequencing. Notwithstanding the extensive genetic prescreening, a conclusive genetic diagnosis could be established in 21 patients (42\%). These included 8 de novo CNVs, including single exon and intraexonic deletions as well as interchromosomal duplications, 12 de novo SNVs affecting the coding region and one compound heterozygous $\mathrm{CNV}$ causing disease in a recessive mode. These results suggest that de novo SNVs and CNVs affecting the coding region are a major cause of severe ID.

\section{Plenary 10 \\ NON-INVASIVE CANCER GENOME SCANNING BY PLASMA DNA ANALYSIS}

\section{Rossa W. K. Chiu}

Li Ka Shing Institute of Health Sciences and Department of Chemical Pathology, The Chinese University of Hong Kong, Hong Kong

Malignant tumors release DNA into plasma. The analysis of tumorassociated nucleic acid markers in plasma enabled the development of blood-based tests for the diagnosis, monitoring and prognostication of cancers. However, cancers are highly heterogeneous in nature. Thus, different biomarkers are required for the detection of cancers of different organs or subtypes. With the advent of nextgeneration sequencing, whereby billions of DNA fragments can be identified and quantified in each analysis run, our group asked if the cancer genome can be decoded directly from human plasma. In our recent studies (Chan et al., Clin Chem 2012; Chan et al., PNAS 2013), we showed that copy number aberrations and methylation profiles of cancers can be detected from plasma DNA sequencing on a genome-wide manner. Because the approach detects any genomic changes that might be associated with a cancer, it can be applied to multiple cancer types. Tumor-associated genomic abnormalities were detected in plasma of patients with cancers of the liver, breast, ovary, lung, colon, nasopharynx or smooth muscles. We analysed plasma samples collected from a patient with synchronous breast and ovarian cancers. The plasma DNA profile revealed collective genetic aberrations from both the breast and ovarian cancers. These data suggest that plasma DNA sequencing is a potential tool for studying tumoral heterogeneity and may allow one to assess the total tumor burden in an individual. The long-term goal of the research is to achieve a test that could be applied for the screening and monitoring of multiple cancer types.

\section{Plenary 11 \\ VARIANT CLASSIFICATION IN HIGH RISK CANCER PREDISPOSITION GENES: THE ROLE OF INTERNATIONAL MULTIDISCIPLINARY CONSORTIA

\author{
Amanda Spurdle $^{1}$, ENIGMA collaborators ${ }^{2}$, InSiGHT collaborators $^{3}$ \\ ${ }^{1}$ QIMR Berghofer Medical Research Institute, Brisbane, QLD, Australia \\ ${ }^{2}$ http://www.enigmaconsortium.org/, Multi-centre, Australia \\ ${ }^{3}$ http://www.insight-group.org/, Multi-centre, Australia
}

Identification of a disease-causing mutation in a cancer syndrome gene directs risk management of carrier individuals, and their relatives - including genetic counseling, pre-symptomatic screening, risk-reducing medication and surgery, and possibly choice of cancer therapy. The BRCA1/2 and mismatch repair (MMR) cancer predisposition genes are commonly tested in the clinical setting Many sequence changes identified by clinical testing are not obviously disease-causing; for example, missense changes, small in-frame insertions/deletions, potential splice site alterations. It is challenging for individual testing laboratories to collect enough information for robust classification of such variants.

Large-scale studies evaluating cancer gene variants in the BRCA and MMR genes are being undertaken with international 
organizations ENIGMA (BRCA1/2) and InSiGHT (MMR). Variant nomenclature and supporting clinical and laboratory information to inform classification has been collated for 'unclassified' sequence variants from clinical testing and research laboratories. Data curation identified considerable inconsistency in documentation of variant nomenclature and variant classification, poor recognition of existing publically available information on variant classification, and need to publicize and implement uniform criteria for classification. Collation and generation of clinical and laboratory information has standardized and promoted variant classification. Areas highlighted for future research include a need to conduct extensive quantitative mRNA/function analysis and family studies to assess the correlation of level and type of splicing/functional aberration with risk.

Results to date demonstrate the value of international collaborative studies to facilitate standardized evidence-based classification of cancer gene variants to improve clinical genetic counseling, and patient and family management.

\section{Plenary 12}

MAPPING OF SIGNIFICANT MUTATIONS IN COMMON CANCERS

David Bowtell ${ }^{1,2}$

${ }^{1}$ Cancer Genomics and Genetics Program, Peter MacCallum Cancer Centre, Melbourne, VIC, Australia

${ }^{2}$ Department of Pathology and Department of Biochemistry, University of Melbourne, Melbourne, VIC, Australia

The development of high throughput technologies, especially next generation DNA sequencing, means that within the next $2-3$ years a complete 'parts list' for human cancer will be available. Collaborative studies, particularly the International Cancer Genome Consortium (ICGC) and The Cancer Genome Atlas (TCGA) are uncovering driver mutations present in human cancer that are detectable at the level of point mutation, changes in DNA copy number, structural variation, transcriptional change, and/or altered pattern of methylation. Genomic analysis of human cancer is shifting from individual tumor types to pan-cancer analyses of thousands of samples across histotypes, identifying processes common to anatomically distinct cancers and resulting in a reclassification of cancer. The presentation will focus on our work on ovarian cancer and cancers of known primary. For the ovarian studies, which are part of the ICGC, I will outline findings from whole genome sequence analysis of over 100 high grade serous cancer genomes. The cancer of unknown primary work is heavily translational and is aimed at improving the clinical management, both through identification of likely site of origin and use of mutational profiling to guide cancer care.

\section{Sutherland Lecture \\ SPLASHING AROUND IN THE GENE POOL: DON'T FORGET THE PATIENT!}

John Christodoulou ${ }^{1,2}$

${ }^{1}$ The Children's Hospital at Westmead, Sydney, NSW, Australia

${ }^{2}$ University of Sydney, Sydney, NSW, Australia

\section{Plenary 13 \\ GENETICS AND GENOMICS TO ELUCIDATE CAUSES OF RARE AND COMMON DISEASES: HOLOPROSENCEPHALY AS AN EXAMPLE}

Maximilian Muenke

National Human Genome Research Institute, Bethesda, MD, USA

\section{Plenary 14}

\section{GENOFACES AND THE ROAD TO UNEMPLOYMENT}

Gareth Baynam $^{1,2}$, Mark Walters ${ }^{5}$, David Gillett ${ }^{5}$, Peter Claes ${ }^{7}$, Mark Shriver ${ }^{6}$, Peter LeSouef $^{2}$, Stefanie Kung ${ }^{2}$, Lyn Schofield ${ }^{1,8}$, Matthew Bellgard ${ }^{8}$, Hugh Dawkins $^{4}$, Jack Goldblatt'

${ }^{I}$ Genetic Services of Western Australia, Perth, WA, Australia

${ }^{2}$ School of Paediatrics and Child Health, University of Western Australia, Perth, WA, Australia

${ }^{3}$ Institute for Immunology and Infectious Diseases, Murdoch University, Perth, WA, Australia

${ }^{4}$ Office of Population Health Genomics, Public Health and Clinical Services Division, Department of Health, Perth, WA, Australia

${ }^{5}$ Craniomaxillofacial Unit, Princess Margaret Hospital, Perth, WA, Australia

${ }^{6}$ Department of Anthropology, Penn State University, PA, USA

${ }^{7}$ Medical Image Computing, KU Leuven, Leuven, Belgium

${ }^{8}$ Centre for Comparative Genomics, Murdoch University, Perth, WA, Australia

Herein we present progress in non-invasive and non-irradiating 3D facial analysis that provides deeply precise objective measurements with a view to enabling clinical unmet need in diagnosis and treatment monitoring. We focus on studies in our, and collaborating, group(s), including novel approaches to asymmetry assessment in syndromic diseases, new facial signatures of rare diseases, and treatment monitoring in metabolic and non-metabolic disorders. We also outline studies exploring the overlap between normal range and syndromic facial variation and foundation methods for converting 3D facial information to text, namely elements of morphology terms. The later may facilitate automated reporting and fusion with text mining approaches for exploring human disease biology and aiding with computer assisted diagnostics.

\section{Plenary 15 \\ THE IDENTIFICATION OF GENE MUTATIONS IN CRANIOSYNOSTOSIS AND THEIR RELATION TO PHENOTYPE AND SURGICAL MANAGEMENT: FROM OCTAVE CROUZON TO MASSIVELY PARALLEL SEQUENCING}

Tony Roscioli', George Elakis ${ }^{2}$, Eric Lee ${ }^{2}$, Timothy Cox ${ }^{3}$, David Moon ${ }^{4}$, Mathew Wallis $^{1}$, Peter Anderson ${ }^{5}$, David David ${ }^{5}$, Anne Turner' ${ }^{1}$, Mark Gianoutsos ${ }^{4}$, Eric $\mathrm{Haan}^{6}$, and Michael F. Buckley ${ }^{2}$

\footnotetext{
${ }^{I}$ Department of Medical Genetics, Sydney Children's Hospital, Sydney, NSW, Australia 2 SEALS Genetics Laboratories, New South Wales Health Pathology, Sydney, NSW, Australia

${ }^{3}$ Seattle Children's Research Institute \& Division of Craniofacial Medicine Department of Pediatrics, University of Washington, Seattle, WA, USA

${ }^{4}$ Craniofacial Unit, Sydney Children's Hospital, Sydney, NSW, Australia

${ }_{5}^{5}$ Australian Craniofacial Unit, Women and Children's Hospital, Adelaide, SA Australia

${ }^{6}$ SA Clinical Genetics Service, SA Pathology at Women Children's Hospital, and Department of Paediatrics, University of Adelaide, Adelaide, SA, Australia
}

Craniosynostosis is one of the most common craniofacial disorders encountered in clinical genetics practice. Between $30-70 \%$ of syndromic craniosynostoses are caused by mutations in hotspots in the fibroblast growth factor receptor (FGFR) genes or in the TWIST1 genes. Here we present results from molecular testing of an Australia and New Zealand cohort of 630 individuals with a diagnosis of craniosynostosis. Data were obtained by Sanger sequencing of FGFR1-3 hotspot exons and the TWIST1 gene, as well as copy number detection of TWIST1. Of the 630 probands, there were 231 who had one of 80 distinct mutations (36\%). Among the $80 \mathrm{mu}-$ tations, 17 novel sequence variants were detected in three of the four genes screened. In addition to the proband cohort there were 96 individuals who underwent predictive or prenatal testing as part 
of family studies. Dysmorphic features consistent with the known FGFR1-3/TWIST1-associated syndromes were predictive for mutation detection. We also show a statistically significant association between splice site mutations in FGFR2 and a clinical diagnosis of Pfeiffer syndrome, more severe clinical phenotypes associated with $F G F R 2$ exon 10 versus exon 8 mutations, and more frequent surgical procedures in the presence of a pathogenic mutation. Although targeting gene hot spot areas for mutation analysis has been a useful strategy to maximize the success of molecular diagnosis for individuals with craniosynostosis, technological developments have allowed additional genes to be sequenced rapidly. We present the initial findings of a massively parallel sequencing gene panel in this cohort, which has extended molecular findings in mutation negative patients.

\section{Plenary 16 \\ AN UPDATE ON CRANIOFACIAL SURGICAL TECHNIQUES AND LONG-TERM RESULTS}

\section{David David}

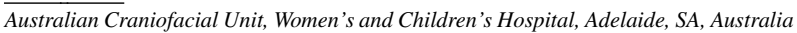

\section{HGSA Oration \\ GENETICS - IT'S PERSONAL}

Eric Haan

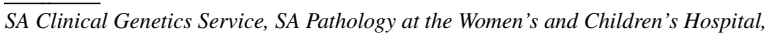
Adelaide, SA, Australia

\section{Plenary 17 \\ ANTISENSE MEDIATED EXON SELECTION TO TREAT HUMAN DISEASE}

\section{Sue Fletcher}

Centre for Comparative Genomics, Murdoch University, Perth, WA, Australia

Alternative exon selection occurs both naturally, increasing the diversity of gene expression, and as a consequence of genetic changes. $\mathrm{We}$, and others, have used antisense oligomers to manipulate both normal and aberrant splicing to alter the course of disease in animal models and in clinical studies. Antisense oligomers can suppress exon selection, or can anneal to splicing silencers to enhance inclusion of exons that are otherwise excluded as a consequence of poor exon definition.

The most advanced antisense oligomer-mediated study at this time is an exon-skipping program using the morpholino oligomer, Eteplirsen to address frame-shifting deletions that flank exon 51 of the dystrophin gene. Such mutations cause the fatal X-linked muscle wasting disease, Duchenne muscular dystrophy. The majority of mutations causing this disease are deletions of one of more exons that disrupt the reading frame, and removal of flanking exon(s) during pre-mRNA processing can reframe the transcript, leading to translation of partially functional dystrophin. Similarly, targeted exclusion of selected exons bearing premature termination codons or indels, or those caused by cryptic splice site activation, can restore the open reading frame and dystrophin expression. An extended phase $2 \mathrm{~b}$ clinical study has shown that exon skipping strategies can maintain ambulation and improve respiratory function in Duchenne muscular dystrophy patients, an outcome that is unprecedented in the history of this disease.
Concurrent 1: Australasian Association of Clinical Geneticists Oral Presentations

\section{AACG Oral 1 \\ APPLICATION OF NGS TO IMPROVING DIAGNOSTIC YIELD FOR INFANTILE-ONSET EPILEPTIC ENCEPHALOPATHIES}

\author{
Elizabeth Palmer ${ }^{1,5}$, M Crowley $^{2}$, Tejaswi Kandula ${ }^{3}$, Annie Bye ${ }^{3,5}$, Marcel \\ Dinger $^{2}$, Eric Lee ${ }^{4}$, Michael F Buckley ${ }^{4}$, Rebecca Macintosh', Rani Sachdev 1,5, \\ Tony Roscioli ${ }^{1,5}$, Edwin Kirk ${ }^{1,5}$ \\ ${ }^{1}$ Department of Medical Genetics, Sydney Children's Hospital, Sydney, NSW, Australia \\ 2 The Kinghorn Center for Clinical Genomics, Garvan Institute, Sydney, NSW, Australia \\ ${ }^{3}$ Department of Neurology, Sydney Children's Hospital, Sydney, NSW, Australia \\ ${ }^{4}$ SEALS Genetics Laboratory, Prince of Wales Hospital, Sydney, NSW, Australia \\ ${ }_{5}^{5}$ School of Women's and Children's Health, University of New South Wales, Sydney \\ NSW, Australia
}

Epileptic encephalopathies (EEs) are characterized by severe, frequent seizures with a detrimental effect on development. The majority of EEs have a genetic basis, with significant genetic heterogeneity (>300 genes). A small subset are treatable. Rapid investigation of children with EE is mandatory to guide therapeutic choices and reproductive counseling. To date, the investigative process requires invasive neurometabolic procedures and financially prohibitive sequential genetic testing, with a low diagnostic yield $(<10 \%)$. Nextgeneration-sequencing (NGS) provides a compelling tool to explore the genomic basis of undiagnosed EE. Recent literature has demonstrated the utility of NGS in epilepsy diagnostics with a diagnostic yield of up to $50 \%$. However, many studies have a paucity of phenotypic information limiting genotype-phenotype correlations and clinical prognostication.

We investigated a cohort of children affected with infantile-onset $\mathrm{EE}$, categorized by clinical and electrophysiological criteria. Whole exome sequencing was performed on 10 affected children using the HiSeq 2500 platform. We performed short read sequence alignment using BWA, and variant calling using the GATK HaplotypeCaller following best practices approaches. Potential causative variants were identified by a tiered approach: (1) comparison with a list of EE genes, (2) comparison of all potentially pathogenic variants with current literature, (3) trio analysis to identify de novo variants. Sanger sequencing was used to confirm candidate mutations. We identified pathogenic/likely pathogenic variants in 5 out of 10 children, including a variant in a newly described EE gene. This study highlights the importance of NGS in Mendelian disorders as a rapid diagnostic technique.

\section{AACG Oral 2 \\ COMPARATIVE HEATH ECONOMIC COSTINGS OF NEXT GENERATION SEQUENCING VERSUS TRADITIONAL NEUROMETABOLIC INVESTIGATION IN A COHORT OF CHILDREN WITH EPILEPTIC ENCEPHALOPATHY.}

Elizabeth Palmer ${ }^{1,5}$, Tejaswi Kandula ${ }^{2}$, Annie Bye $^{2,5}$, M Crowley $^{3}$, Marcel Dinger $^{3}$, Erik Lee ${ }^{4}$, Edwin Kirk ${ }^{1,5}$, Michael F Buckley ${ }^{4}$, Rani Sachdev 1,5 , Tony Roscioli ${ }^{1,5}$

\footnotetext{
${ }_{1}^{1}$ Department of Medical Genetics, Sydney Children's Hospital, Sydney, NSW, Australia ${ }^{2}$ Department of Neurology, Sydney Children's Hospital, Sydney, NSW, Australia

${ }^{3}$ The Kinghorn Center for Clinical Genomics, Garvan Institute, Sydney, NSW, Australia ${ }^{4}$ SEALS Genetics Laboratory, Prince of Wales Hospital, Sydney, NSW, Australia 5 School of Women's and Children's Health, University of New South Wales, Sydney, NSW, Australia
}

Objective: Epileptic encephalopathy (EE) presents with severe seizures associated with developmental delay. A subset has effective treatments, and therefore rapid diagnosis is a priority to guide management. The investigation of children with EE is complex but the 
majority have a genetic basis, with several hundred genes known. The cost of the investigation of children with $\mathrm{EE}$ is immense as the results of neurometabolic and genetic disorders often display phenotypic overlap with other diseases. Numerous invasive investigations invariably include MRI scanning (with general anaesthesia), multiple EEGs, lumbar puncture for CSF metabolites and extensive serum/urine neurometabolic testing. Individual molecular genetic tests have a low sensitivity. Identifying specific gene mutations has been challenging until the advent of next generation sequencing (NGS). The current revolution in diagnostic molecular genetics through NGS now allows the testing of all genes in a cost-effective and timely fashion. We will investigate whether a genomic diagnostic approach to children with Epileptic Encephalopathy (EE) is a viable alternative to the traditional diagnostic algorithm. Design and Participants: A retrospective observational study of a cohort of 10 patients affected with EE has been performed. All patients have undergone the traditional neurometabolic investigative approach and were subsequently tested with NGS. With a health economist we have examined the cost-effectiveness of NGS to determine whether it is a viable, rapid, high-yield and a less expensive alternative to current sequential investigations. We propose, through these results, that NGS provides a comprehensive and more cost-effective management scheme for the investigation of EE.

\section{AACG Oral 3 \\ NEXT GENERATION SEQUENCING PROVIDES ANSWERS FOR FAMILIES AFFECTED BY FOETAL AKINESIA, ARTHROGRYPOSIS, AND SEVERE CONGENITAL MYOPATHIES}

Emily Todd', Kyle Yau', Royston Ong', Jennie Slee ${ }^{2}$, George McGillivray ${ }^{3}$, Cathy Kiraly-Borri ${ }^{4}$, Elizabeth Thompson ${ }^{5,6}$, Christopher Barnett ${ }^{5,6}$, Goknur Haliloglu $^{7}$, Ariana Kariminejad ${ }^{8}$, Caroline Sewry ${ }^{9}$, Anita Cairns ${ }^{10}$, Nigel Clarke $^{11,12}$, Alison Colley ${ }^{13}$, Monique Ryan ${ }^{14}$, Padma Sivadorai ${ }^{15}$, Mark Davis ${ }^{15}$, Richard Allcock ${ }^{16}$, Nigel Laing ${ }^{1,15}$, Gianina Ravenscroft ${ }^{1}$

${ }^{1}$ Harry Perkins Institute of Medical Research and the Centre for Medical Research, University of Western Australia, Perth, WA, Australia

${ }^{2}$ Genetic Services of Western Australia, King Edward Memorial Hospital, Perth, WA, Australia

${ }^{3}$ Victorian Clinical Genetics Services, Murdoch Childrens Research Institute, The Royal Children's Hospital, Melbourne, VIC, Australia

${ }^{4}$ Genetic Services of Western Australia, Princess Margaret Hospital for Children and King Edward Memorial Hospital, Perth, WA, Australia

${ }^{5}$ Department of Paediatrics, University of Adelaide, Adelaide, SA, Australia

${ }^{6}$ Paediatric and Reproductive Genetics Unit, SA Clinical Genetics Service, SA Pathology at the Women's and Children's Hospital, Adelaide, SA, Australia

7 Department of Pediatric Neurology, Hacettepe University Children's Hospital Ankara, Turkey

${ }^{8}$ Kariminejad-Najmabadi Pathology and Genetics Centre, Tehran, Iran

${ }^{9}$ Dubowitz Neuromuscular Centre. Institute of Child Health and Great Ormond Street

Hospital, London, UK

${ }^{10}$ Mater Misericordiae Health Services, Mater Children's Hospital, Brisbane, QLD, Australia

${ }^{11}$ Institute for Neuroscience and Muscle Research, The Children's Hospital at

Westmead, Sydney, NSW, Australia

${ }_{12}$ Australia and the Discipline of Paediatrics and Child Health, University of Sydney, Sydney, NSW, Australia,

${ }^{13}$ Department of Clinical Genetics, South Western Sydney Local Health District, Sydney, NSW, Australia

${ }_{14}$ Department of Neurology, The Royal Children's Hospital, Melbourne, VIC, Australia

15 Department of Molecular Genetics, Pathwest, QEII Medical Centre, Perth, WA, Australia

${ }^{16}$ Lotterywest State Biomedical Facility Genomics and School of Pathology and Laboratory Medicine, University of Western Australia, Perth, WA, Australia

The fetal akinesia syndromes, severe congenital myopathies, and arthrogryposes are all heterogeneous disorders with prenatal or neonatal onset. Despite several causative genes being associated with these disorders, many cases remain without a genetic diagnosis. Next generation sequencing (NGS), can identify mutations in known and novel disease genes. Genomic DNA from individuals from 40 unrelated families with fetal akinesia, a severe congenital myopathy or arthrogryposis, was subjected to NGS with the aim of identifying the genetic cause of disease. Individuals were subjected to either whole exome sequencing or a custom-designed supercapture array, consisting of 277 known neuromuscular disease genes. Variants fitting the phenotype and inheritance pattern were investigated, and confirmed using Sanger sequencing.

Analysis of NGS data resulted in a genetic diagnosis for 17/40 families, including 3/14 affected by a fetal akinesia syndrome, 9/15 by a severe congenital myopathy, and 5 of 11 with arthrogryposis. Mutations were identified in eight different known neuromuscular disease genes (CHRND, CHRNG, ECEL1, GBE1, MTM1, $M Y H 3, N E B$, and $R Y R 1)$ and three novel neuromuscular disease genes (KLHL4O, KLHL41, and Gene X). In conclusion, the use of NGS on a cohort of families with fetal akinesia, a severe congenital myopathy or arthrogryposis resulted in a diagnosis in $42 \%$ of cases. This study highlights the capabilities of NGS in determining the genetic diagnosis in heterogeneous diseases, as well as the ability to identify novel disease genes. Finally, this study has expanded the genotype-phenotype correlations for known neuromuscular disease genes.

\section{AACG Oral 4 \\ PERIVENTRICULAR NEURONAL HETEROTOPIA CAUSED BY MUTATIONS AFFECTING THE DIMERIZATION DOMAIN OF FLNA: GENOTYPE: PHENOTYPE CORRELATIONS}

Margriet van Kogelenberg' ${ }^{\text {, Alice } \text { Clark }^{2} \text {, Andrew Sutherland-Smith }}{ }^{2}$, Matt Edwards $^{3}$, Tracy Dudding ${ }^{3}$, Stephen Robertson ', Ananda Anandan ${ }^{2}$

${ }^{1}$ University of Otago, Dunedin, New Zealand

${ }^{2}$ Insitute of BioSciences, Massey University, Palmerston North, New Zealand

${ }^{3}$ Hunter Genetics, Newcastle, NSW, Australia

Filamin A, the filamentous protein encoded by the X-linked gene FLNA, crosslinks cytoskeletal actin into three dimensional networks, facilitating its role as a signaling scaffold and a mechanosensor of extrinsic shear forces. Central to these functions is the ability of FLNA to form V-shaped homodimers through its C-terminal located filamin repeat 24 . Two brothers with mild periventricular heterotopia $(\mathrm{PH})$, a neuronal migration disorder typically caused by loss-of-function mutations in FLNA, are described with a missense mutation (p.Gly2593Glu) inserting a large negatively charged amino acid into the hydrophobic dimerization interface of FLNA. Additional mutations in the same vicinity in four unrelated individuals conferred a more typical presentation of $\mathrm{PH}$ in female heterozygotes and presumptive lethality in hemizygous males, further underscoring the exceptionally mild consequences of the p.Gly2593Glu substitution. Co-immunoprecipitation, in vitro cross-linking studies and gel filtration chromatography all demonstrated that homodimerization of isolated FLNA repeat 24 is abolished by this Gly2593Glu substitution but that extended FLNA ${ }^{\text {Gly2593Glu }}$ repeat 16-24 constructs exhibit dimerization . Collectively these observations imply that other interactions apart from that mediated by the canonical repeat 24 dimerization interface can help mediate FLNA homodimerization. 
Concurrent 2: Australasian Society of Genetic Counsellors Oral Presentations

\section{ASGC Oral 1 \\ PSYCHOLOGICAL IMPACT OF INCONCLUSIVE BRCA1/2 TEST RESULTS}

Jennifer Berkman', Ruth Simpson ${ }^{2}$, Jane Fleming', Madelyn Peterson ${ }^{2}$

${ }^{I}$ Genetic Health Queensland, Royal Brisbane and Women's Hospital, Brisbane, QLD, Australia

${ }^{2}$ Department of Biomolecular and Physical Sciences, Griffith University, Brisbane, $Q L D$, Australia

Individuals with breast and/or ovarian cancer who undergo diagnostic testing for BRCA1 and BRCA2 gene mutations can receive three possible results: a pathogenic mutation is found; no mutation is found (NMF); or an unclassified variant (UCV) is detected. For those with NMF or UCV results, the empiric cancer risks for relatives are unaltered. To investigate patient understanding of these results and their psychological impact, a questionnaire was sent to 200 Genetic Health Queensland patients who received NMF and UCV test results between 2008 and 2011. Seventy questionnaires were returned. Results showed that the majority of participants did not understand their results, but this did not negatively impact on their screening behavior. Participants were also asked to describe their feelings upon receipt of their results and to comment on why they felt this way. The majority (49/70) of participants felt relieved and/or satisfied with their test result. While these emotions are understandable, the reasons given for feeling relieved were concerning in some cases. Seven of 34 participants who were relieved stated that they were relieved that their daughters or relatives would not get breast cancer, or were not at increased risk of breast cancer. It is therefore likely that relatives in some families have been told that they are not at increased risk of breast cancer, which may lead to reduced surveillance. This suggests a need for improved communication of these results for some patients. This and other identified psychosocial issues associated with inconclusive test results are presented. support group has played in their dealing with their mutation status and how it has influenced their decision making. From this we aim to identify factors that have contributed to the longevity and perceived success of this particular group, as well as the disadvantages, in order to guide the establishment of future support groups. We seek to study why this group has been successful and how this success can be replicated in other support groups. These results will then be used as a basis for further care and research, specifically in the development and evaluation of a future support group, to be formed in $2014 / 2015$.

\section{ASGC Oral 3 \\ Ron Fleischer $^{1,2}$, Lisa Bristowe ${ }^{1,3}$ \\ ${ }^{1}$ The Genetic Clinic, Sydney, NSW, Australia \\ 2 Royal Prince Alfred Hospital, Sydney, NSW, Australia \\ 3 Sydney Children's Hospital, Sydney, NSW, Australia}

PRECONCEPTION GENETIC SCREENING: REFLECTIONS ON THE FIRST 26 PATIENTS TO UNDERGO THE COUNSYL TEST IN A PRIVATE GENETIC COUNSELING SETTING.

The Counsyl Universal Genetic Test is a commercially available genetic test that screens for up to 417 disease-causing mutations associated with up to 108 genetic disorders. It includes cystic fibrosis, spinal muscular atrophy, Fragile $\mathrm{X}$ syndrome, beta thalassemia, Tay-Sachs disease and a large number of rarer, autosomal recessive disorders. It is not a direct-to-consumer test and must be ordered through a genetic counselor or doctor.

We offer this test in a private genetic counseling setting where patients pay a standard fee for a consultation and then pay for any additional pathology directly to the pathology provider.

In our first 26 patients who requested Counsyl testing, we identified 13 carriers. Two were carriers for two conditions. There was one test that failed. One couple were suspected to be epidermolysis bullosa carriers and this was confirmed; one couple were hypothesized to be Connexin 26 carriers and this was also confirmed.

In a series of vignettes, we will highlight the genetic counseling challenges of offering the Counsyl Universal Genetic Test and reflect on the demographics and motivations of patients requesting this test. All patients had a heightened awareness of the risk of having a child with a disability, and saw the Counsyl test as a way to mitigate this risk.

\section{ASGC Oral 4}

EXPLORING THE PSYCHOSOCIAL SUPPORT SERVICES FOR WOMEN WITH DIVERGENCE OF SEX DEVELOPMENT

Chloe Hanna' ', A/Prof Sonia Grover ${ }^{1,4}$, Louisa Di Pietro ${ }^{2,5}$, Melody Meneszes ${ }^{2,3}$

\footnotetext{
University of Melbourne, Melbourne, VIC, Australia

Murdoch Children's Research Institute, Melbourne, VIC, Australia

${ }^{3}$ Monash Ultrasound for Women, Melbourne, VIC, Australia

${ }^{4}$ Royal Children's Hospital, Melbourne, VIC, Australia

Victorian Clinical Genetic Services, Melbourne, VIC, Australia
}

Women living with disorders of sex development (DSD) may have both complex genetic differences and gynaecological features which have an impact on their reproductive health. These differences can also impact on fertility, body image, self-esteem and social acceptance. This qualitative study aimed to explore the psychosocial support needs of women who have a DSD. Ten semi-structured interviews were conducted with women diagnosed with a DSD aged between 21 to 34 years. Participants were asked to discuss their wishes, needs and expectations of support services. Interviews were audiotaped, transcribed verbatim and analysed using thematic analysis. 
Findings revealed that overall participants felt satisfied with the available support systems, chiefly family support. However, social acceptance, formation of intimate relationships, coping with the physical implications, understanding reproductive options and maintaining better health outcomes were identified by participants as issues that could be improved with psychosocial support. Professional psychosocial and peer support services were identified as essential components of condition management and participants felt these should be offered as part of standard care practice. The majority of participants highlighted the value of appropriate peer relationships and wanted more support in this area. Participants recommended the development and provision of a resource inclusive of up-to-date health information, advice on fertility pathways, and contact information for both medical, psychosocial and peer support.

Concurrent 3: Australasian Society of Inborn Errors of Metabolism Oral Presentations

\section{ASIEM Oral 1 MUTATIONS IN LYRM4, ENCODING IRON-SULFUR CLUSTER BIOGENESIS FACTOR ISD11, CAUSE DEFICIENCY OF MULTIPLE RESPIRATORY CHAIN COMPLEXES \\ David Thorburn $^{1,3}$, Sze Chern Lim ${ }^{1,3}$, Martin Friemel ${ }^{4}$, Justine Marum ${ }^{1,3}$, Elena Tucker ${ }^{1,3}$, Damien Bruno ${ }^{2}$, Lisa Riley ${ }^{5,6}$, John Christodoulou ${ }^{5,6}$, Edwin Kirk ${ }^{7}$, Avihu Boneh ${ }^{2,3}$, Tracey Rouault ${ }^{8}$, Silke Leimkühler ${ }^{4}$, Alison Compton' \\ ${ }^{I}$ Murdoch Children's Research Institute, Melbourne, VIC, Australia \\ 2 Victorian Clinical Genetics Services, Melbourne, VIC, Australia \\ ${ }^{3}$ University of Melbourne, Melbourne, VIC, Australia \\ ${ }^{4}$ University of Potsdam, Potsdam, Germany \\ ${ }^{5}$ Children's Hospital at Westmead, Sydney, NSW, Australia \\ ${ }^{6}$ University of Sydney, Sydney, NSW, Australia \\ 7 Sydney Children's Hospital, Sydney, NSW, Australia \\ ${ }^{8}$ Eunice Kennedy Shriver National Institute of Child Health and Human Development, Bethesda, MD, USA}

We previously reported sequencing $\sim 1000$ genes encoding the known mitochondrial proteome in 42 infants with definite biochemical OXPHOS disorders (Calvo et al., 2012 Sci Trans Med 4:118ra10). In addition to proven diagnoses, this identified mutations in a number of candidate disease genes. A patient with deficiency of complexes I, II and III in muscle and liver had a homozygous mutation (c.203G > T, p.R68L) in LYRM4, encoding the ISD11 protein. ISD1 1 forms a complex with the sulfur donor NFS1 and stabilizes it. Complexes I, II and III rely on iron sulfur (Fe-S) clusters for enzyme activity. Sanger sequencing identified the same mutation in his similarly affected cousin, who had a more severe phenotype, died in the neonatal period and had deficiency of Complex IV in addition to Complexes I, II and III defects. Other Fe-S proteins were also affected in both patients, including the aconitases and ferrochelatase. Mutant ISD11 only partially complemented for an ISD11 deletion in yeast. In vitro studies showed that the L-cysteine desulfurase activity of NFS1 was barely present when co-expressed with mutant ISD11. LYRM4 mutations cause a novel defect in an early step of $\mathrm{Fe}-\mathrm{S}$ cluster assembly, affecting a broad variety of Fe-S proteins. The differences in biochemical and clinical features between the two patients may relate to limited availability of cysteine in the newborn period and suggest a potential approach to therapy.

\section{ASIEM Oral 2 \\ WHOLE EXOME SEQUENCING CONFIRMS LEIGH SYNDROME IN A PATIENT SHOWING LITTLE BIOCHEMICAL EVIDENCE OF A MITOCHONDRIAL DISORDER}

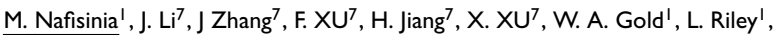
D.-R. Thorburn ${ }^{4}$, B. Keating ${ }^{3}$, Y. Guo ${ }^{3}$, H. Hakonarson ${ }^{3}$, J. Christodoulou 9

${ }^{1}$ Genetic Metabolic Disorders Research Unit, Western Sydney Genetics Program, The Children's Hospital at Westmead, Sydney, NSW, Australia

${ }^{2}$ Discipline of Paediatrics and Child Health, Sydney Medical School, University of Sydney, Sydney, NSW, Australia

${ }^{3}$ Centre of Applied Genomics, The Children's Hospital of Philadelphia, PA, USA

${ }^{4}$ Murdoch Children's Research Institute and Victorian Clinical Genetics Services, Royal Children's Hospital, Melbourne, VIC, Australia

${ }^{5}$ Department of Paediatrics, University of Melbourne, Melbourne, VIC, Australia

${ }^{6}$ The Institute for Neuromuscular Research, The Children's Hospital at Westmead, Sydney, NSW, Australia

${ }^{7}$ Institute of Botany, Chinese Academy of Sciences, Beijing, China

${ }^{8}$ Shenzhen Key Laboratory of Transomics Biotechnologies, Shenzhen, China

${ }^{9}$ Discipline of Genetic Medicine, Sydney Medical School, University of Sydney, Sydney, NSW, Australia

Background: Leigh syndrome is a severe neurological disorder caused by mutations in one of more than 30 genes; most of which are associated with the mitochondrial respiratory chain (RC). Aim: To identify the genetic cause of disease in a patient with an overall clinical picture of Leigh syndrome. Patient and Methods: A girl with a clinically suspected diagnosis of Leigh syndrome was born to healthy and non-consanguineous parents after a normal pregnancy and delivery. She was first hospitalized at 2 years of age for febrile seizures and partial left ptosis. She then presented with left-sided weakness of her arm and leg, scoliosis and worsening dystonia at 6 years. Magnetic resonance imaging of her brain showed symmetrical putaminal abnormalities. Her lactic acid level was elevated on brain magnetic resonance spectroscopy but normal in blood and cerebrospinal fluid. Whole exome sequencing was used to screen for likely pathogenic variations in the patient followed by mitochondrial enzyme assay and Western blotting. Results: Wholeexome sequencing uncovered compound heterozygous mutations in the NADH dehydrogenase ubiquinone flavoprotein 1 [NDUFV1] (c.1162+4A $>$ C, resulting in skipping of exon 8, and c.G640A, p.Glu214Lys), both previously associated with complex I deficiency and Leigh syndrome. Despite normal complex I enzyme activity in patient muscle, liver and fibroblasts, the protein level of NDUFV1 was significantly reduced by $75 \%$ on Western blotting. Conclusion: Whole exome sequencing can be used to provide a definitive diagnosis of a suspected mitochondrial RC disorder even in cases where $\mathrm{RC}$ enzyme activity is apparently normal.

\section{ASIEM Oral 3 \\ AGRESSIVE DIETARY MANAGEMENT DRAMATICALLY IMPROVES MARKED TRANSAMINITIS AND CLINICAL PROFILE IN GLYCOGEN STORAGE DISEASE TYPE IXA}

Shanti Balasubramaniam', Beth Martino ${ }^{3}$, Andrew Martin', Geoff Knight', Greed Lawrence ${ }^{4}$, Leah Queit

${ }^{1}$ Metabolic Unit, Department of Paediatrics and Adolescent Medicine, Princess Margaret Hospital, Perth, WA, Australia

${ }^{2}$ University of Western Australia, Perth, WA, Australia

${ }^{3}$ Dietetic Department, Princess Margaret Hospital, Perth, WA, Australia

${ }^{4}$ PathWest Laboratories WA, Princess Margaret Hospital, Perth, WA, Australia

The purpose of this report is to describe an atypical, severe presentation of glycogen storage disease (GSD) IXa and the aggressive dietary management used to dramatically improve metabolic control. GSD IXa (resulting from PHKA2 mutations) has been widely 
regarded as a benign condition that does not warrant treatment because the childhood symptoms frequently improve with age. Patients present in childhood with hepatomegaly, short stature and ketotic hypoglycaemia. Long term complications including hepatic fibrosis or cirrhosis have only been reported recently. We present a 3-yearold boy with GSD IXa who had a massively enlarged liver with fibrosis and deranged biochemistry with hypoglycaemia and ketosis at the time of diagnosis. Structured dietary interventions including regular daytime and continuous overnight feeds, frequent doses of uncooked cornstarch and protein supplementation was initiated. Diagnostic confirmation of the disorder was performed by mutational analysis of the PHKA2 gene. Regular liver function tests, home blood glucose and ketones monitoring and semi-quantitative estimation of the liver size were used to access clinical improvement. Considerable clinical and biochemical improvements including enhanced growth velocity, energy levels, overall well-being, decreased hepatomegaly, rapidly improved liver enzymes and stabilized blood glucose and ketone levels were demonstrated with the initiation of aggressive dietary therapy. These results concur with a recent report in 2013 by Tsilianidis and colleagues who proposed that GSD IX $\alpha$ is not always a mild condition, but instead part of an expanding phenotypic spectrum. Consideration of intensive dietary interventions is imperative to improve quality of life in these patients.
Concurrent 4/5: Australasian Society of Cytogeneticists and Molecular Genetics Society of Australasia Oral Presentations

\section{ASoC \& MGSA Oral 1/2 NON-INVASIVE PRENATAL KARYOTYPING AND FETAL GENOME SCANNING BY MATERNAL PLASMA DNA ANALYSIS}

Rossa Chiu

Li Ka Shing Institute of Health Sciences and Department of Chemical Pathology, The Chinese University of Hong Kong, Hong Kong

The presence of cell-free fetal DNA in the plasma of pregnant women was first reported in 1997. Just over a decade of research later, tests based on maternal plasma DNA analysis achieve fetal chromosomal aneuploidy detection at $99 \%$ accuracy. Since 2011 , such non-invasive tests have been introduced clinically as part of prenatal screening programs in many parts of the world. Recent research has further shown that maternal plasma DNA analysis could be applied to determine the fetal karyotype, scan the fetal genome for genetic variants for the diagnosis of single gene diseases and to determine twin zygosity. In summary, non-invasive prenatal diagnosis has become a clinical reality and resulted in shifts in prenatal practices.

\section{ASIEM Oral 4 \\ SHOULD MALONYL-COA DECARBOXYLASE DEFICIENCY (MCDD) BE CONSIDERED FOR NEWBORN SCREENING? \\ Ricky Price', Brianna Myers', Karen Beadle', Christine Gurnsey', Suzanne Neven $^{2}$, Michael Henman ${ }^{2}$, Avis McWhinney ${ }^{2}$, Tavey Dorofaeff ${ }^{3}$, Anita Inwood ${ }^{3}$, Ming Liew ${ }^{3}$, Frank Bowling ${ }^{4}$, Jim McGill ${ }^{1,2,3}$ \\ ${ }^{1}$ Division of Chemical Pathology, Pathology Queensland, Brisbane, QLD, Australia \\ ${ }^{2}$ Chemical Pathology Mater Pathology, Brisbane, QLD, Australia \\ ${ }^{3}$ Royal Children's Hospital, Brisbane, QLD, Australia \\ ${ }^{4}$ Mater Children's Hospital, Brisbane, QLD, Australia}

Combined malonic and methylmalonic aciduria is most commonly due to MCDD. Some cases are due to acyl-CoA synthetase deficiency (ACSD), a benign disorder. MCDD is not currently included in the newborn screening panels in Australia. Case 1: An 11-monthold boy with a history of failure to thrive and developmental delay, presented to a regional hospital after 5 days of vomiting. He had a metabolic acidosis with $\mathrm{pH} 7.08$ and was retrieved to the $\mathrm{RCH}$ by plane. Urine organic acids showed marked ketoacidosis and malonate $6400>$ methylmalonate $300 \mathrm{mmol} / \mathrm{mol}$ creatinine, typical of MCDD . MRI showed acute changes in the basal ganglia. An echocardiogram showed mild cardiomyopathy. The acidosis improved with $10 \%$ dextrose and intravenous lipids. A high MCT containing formula was introduced. Case 2: This normal 21-month-old boy was admitted with dehydration and hypoglycaemia after vomiting and diarrhea. He recovered quickly with IV fluids. Urine organic acids showed marked ketoacidosis, methylmalonate $902>$ malonate $445 \mathrm{mmol} / \mathrm{mol}$ creatinine. Plasma propionylcarnitine was normal. This was typical of ACSD. A review of our unreported newborn screening data showed an elevated malonylcarnitine (C3DC) from Case 1 but not Case 2. Conclusion: MCDD can be detected by newborn screening. ASCD can be differentiated biochemically. The acute presentation and cerebral insult in Case 1 could have been prevented had the diagnosis been known. Recent evidence shows reversal of cardiomyopathy by a high MCT diet. Although dietary intervention may not prevent the cognitive problems, children with MCDD deficiency would benefit from early recognition by newborn screening.

\section{ASoC \& MGSA Oral 3 DEVELOPING THE RIGHT TEST: DIAGNOSTIC IMPLEMENTATION OF NEXT-GENERATION SEQUENCING}

Karin Kassahn ${ }^{1,2}$

${ }^{1}$ Genetic and Molecular Pathology, SA Pathology, Adelaide, SA, Australia ${ }^{2}$ School of Molecular and Biomedical Sciences, The University of Adelaide, Adelaide, SA, Australia

The implementation of next-generation sequencing in diagnostic laboratories around the country is promising to improve the costeffectiveness of genetic testing and the diagnostic pick-up rate. A number of technology platforms and sequencing strategies are available, ranging from targeted gene panels to whole-exome and wholegenome sequencing. As part of our service development we have been assessing the benefits of different sequencing approaches for application in inherited disorders, cardiomyopathies, familial cancer, somatic mutation detection in tumors and pediatrics. These applications differ in the number of known disease-associated genes, clinically actionable genes, the pick-up rate of existing methods and the sensitivity of detection required. We describe strategies we have employed to choose technology platforms for new test development. Current efforts are concentrating on the validation of new tests and we give examples of our efforts to examine the accuracy and sensitivity of detection. Finally, when expanding our gene-testing service we have encountered challenges in managing the annotation and clinical interpretation of genomic variants. We describe some of the resources and tools we have examined. Finally, as with any new technology, successful clinical implementation depends largely on the successful integration into the clinical management of patients; having chosen the 'right' group of disorders and genes to test after consultation with the key clinical end users and managing clinician information and patient consent and developing appropriate reporting formats. It is an exciting time in medical genetic testing with new technologies and new tests continuously advancing the scope of our services. 


\section{ASoC \& MGSA Oral 4 \\ GENETIC AUTOPSY OF PERINATAL DEATH: DIAGNOSIS AND DISCOVERY BY WHOLE EXOME AND WHOLE GENOME SEQUENCING}

Alicia Byrne ${ }^{1,9}$, Christopher Barnett ${ }^{2,7}$, Lynette Moore ${ }^{3}$, Yee Khong ${ }^{4}$, Sui $\mathrm{Yu}^{5}$, Nathalie Nataren ${ }^{1,6}$, Quenten Schwarz ${ }^{1,7}$, Andreas Schreiber ${ }^{6,8}$, Jinghua Feng ${ }^{6,8}$, Jill Lipsett ${ }^{3}$, Hahn Chris ${ }^{1,7}$, Hamish Scott ${ }^{1,8}$

${ }^{I}$ Department of Molecular Pathology, Centre for Cancer Biology, SA Pathology, Adelaide, SA, Australia

2 SA Clinical Genetics, Women's and Children's Hospital/SA Pathology, Adelaide, SA, Australia

${ }^{3}$ Department of Anatomical Pathology, Women's and Children's Hospital/SA Pathology, Adelaide, SA, Australia

${ }^{4}$ Department of Anatomical Pathology, Royal Adelaide Hospital/SA Pathology, Adelaide, SA, Australia

${ }^{5}$ Department of Genetics and Molecular Pathology, Women's and Children's Hospital/SA Pathology, Adelaide, SA, Australia

${ }^{6}$ School of Molecular and Biomedical Science, University of Adelaide, Adelaide, SA, Australia

${ }^{7}$ School of Medicine, University of Adelaide, Adelaide, SA, Australia

${ }^{8}$ ACRF Cancer Genomics Facility, Centre for Cancer Biology, SA Pathology, Adelaide, SA, Australia

${ }^{9}$ School of Pharmacy and Medical Sciences, University of South Australia, Adelaide, SA, Australia

Next generation sequencing technologies are proving to be powerful tools in the identification of genetic variants underlying rare genetic disorders. Our laboratory has undertaken whole exome sequencing (WES) and whole genome sequencing (WGS) on a number of cases of perinatal death (PD). PD is defined as the death of a fetus after a pregnancy of at least 20 weeks gestation or weighing $400 \mathrm{~g}$, or of a neonate within 28 days of birth. At present, the autopsy of PD requires a complex investigation involving medical imaging, laboratory investigations, and the examination of the body and placenta. Despite such careful process, a cause may not be found in approximately $22 \%$ of cases. We hypothesized that WES or WGS would act as a 'genetic autopsy', providing an explanation for many cases of PD and improving the definitive diagnostic yield. We also expected to identify novel monogenic disease genes with functions critical in embryonic developmental pathways.

The validity of this approach was first demonstrated using WES to identify a novel homozygous variant in FGFR2 causing neonatal lethality in a fetus from consanguineous parents. WGS has been applied to unexplained polycystic kidney disease (PKD) in two fetuses from consanguineous parents, found to share three regions of homozygosity (ROH). No known PKD genes were located within their ROH. WGS and analysis of ROH has identified only 1 outstanding candidate gene. Segregation analysis and primary functional assays are being performed to assess pathogenicity.

Concurrent 6: Australasian Association of Clinical Geneticists Oral Presentations

\section{AACG Oral 5 \\ THE PLIGHT OF A STATE-WIDE GENETIC SERVICE: INCREASING CONSUMER DEMAND AND INTRODUCTION OF ACTIVITY BASED FUNDING}

Anne Hawkins' ${ }^{\text {, Jack Goldblatt }}{ }^{1,2}$, Hugh Dawkins ${ }^{2}$, Caroline Graham ${ }^{2}$

${ }^{1}$ Genetic Services of Western Australia, Perth, WA, Australia

${ }^{2}$ Office of Population Health Genomics, Department of Health WA, Perth, WA, Australia

Genetic Services WA (GSWA) is the single state-wide clinical genetics provider for Western Australia (WA). WA Health is moving to an Activity Based Funding (ABF) model that counts clinical service events (CSE) from inpatient, outpatient and telephone consultations. Extra-clinical activities such as case preparation, follow-up, professional meetings, teaching and research are not captured by CSE. Distribution of resources to serve rural clinics is not adequately captured by CSE. ABF underestimates clinician time required to provide high quality state-wide services for complex rare diseases. The risk of strictly applying the framework is severe underfunding, as evidenced by the reduction of the Independent Hospital Pricing Authority (IHPA) weight applied to tier two clinics from 0.1312 to 0.0497 for fiscal year 2013/2014 from which the consequence to GSWA equated to approximately $70 \%$ loss of activity based revenue. GSWA is working at capacity and cannot increase activity to meet demand without additional resources. During 2012-2013, new referrals received by GSWA increased by $32 \%$. New referrals to the Familial Cancer Program increased by 79\%. In 2012, 24\% of new referrals exceeded GSWA's capacity to provide an appointment within 12 months, and this rose to $41 \%$ in 2013 . The cumulative loss of revenue associated with lost/delayed reportable CSE activity corresponds to at least $\$ 1.8$ million over 24 months. Stagnant FTE levels associated with underfunding by ABF will adversely affect GSWA. GSWA is actively working to raise its profile through business cases and collaboration with other Australian genetic services to guide the path to a sustainable future.

\section{AACG Oral 6 \\ THE QUEENSLAND RENAL GENETICS MDT CLINICAL} SERVICE

Chirag Patel', Andrew Mallet ${ }^{2}$, Helen Healy ${ }^{2}$, Julie McGaughran'

${ }^{1}$ Genetic Health Queensland, Royal Brisbane and Women's Hospital, Brisbane, QLD, Australia

${ }^{2}$ Department of Renal Medicine, Royal Brisbane and Women's Hospital, Brisbane, $Q L D$, Australia

We report the data from the first known Renal Genetics MDT clinical service in Australia, attended by a Clinical Geneticist and Nephrologist. Since August 2013, 30 patients have been seen in the clinic based on referral type: (a) known genetic kidney disease (GKD) disease information/genetic counseling (11), (b) suspected GKD with a family history - diagnostics (9), or (c) suspected GKD without a family history - diagnostics (10). Referrals were from nephrologists (14), medical specialists (2), and GPs (14). Diagnostic categories included: ADPKD (8), non-ADPKD renal cysts (7), nephropathy (6), metabolic (2), syndromic (3), and unknown cause for renal failure (4).

All patients were given information on the diagnosis or differential diagnoses. We delineated a family history in 16 patients $(53 \%)$. Genetic counseling was provided to 21 patients $(70 \%)$. Genetic tests were ordered in 14 patients (47\%), indications being: a) diagnostic (14\%), genetic counseling (14\%), or both $(72 \%)$. Of these, 3 have positive results and 11 are pending. Other non-genetic investigations (imaging/urinary studies) were ordered in $6(20 \%)$ patients for diagnostics.

So far the successful outcomes of the clinic have been: (a) disease information and genetic counseling in one clinic appointment for 21 patients, (b) new clinical diagnoses in 2 patients, (c) new clinical and molecular diagnoses in 2 patients, and (d) molecular confirmation of a clinical diagnosis for genetic counseling in 1 patient. The positive experiences of this clinic model highlight the need for mainstreaming genetics services and the development of similar clinics in locations across Australia. 
AACG Oral 7

\section{A PLAN FOR A NATIONAL POPULATION-BASED PROGRAM} FOR DETECTION OF LYNCH SYNDROME

Nicholas Pachter $^{1,2}$, Lyn Schofield ${ }^{1,4}$, Jack Goldblatt ${ }^{1,2}$, Fabienne Grieu ${ }^{3}$, Benhur Amanuel $^{3}$, Barry lacopetta ${ }^{4}$

${ }^{1}$ Genetic Services of Western Australia, King Edward Memorial Hospital, Perth, WA, Australia

${ }^{2}$ School of Paediatrics and Child Health, University of Western Australia, Perth, WA, Australia

${ }^{3}$ Molecular Anatomical Pathology, PathWest QEII, Perth, WA, Australia

${ }^{4}$ School of Surgery, University of Western Australia, Perth, WA, Australia

While there is good evidence to support the systematic screening of colorectal cancer cases at diagnosis for Lynch syndrome, its implementation in various parts of Australia is inconsistent. We have previously established, in a large retrospective study in Western Australia, that testing for microsatellite instability in colorectal cancers from patients aged $<60$ years was an effective initial screen to identify individuals with LS. From these findings, MSI and/or mismatch repair protein immunohistochemical screening was recommended for all newly diagnosed CRC patients aged $<60$ years in WA, regardless of a family history of cancer.

Four years experience with such testing (Jan 2009-December 2012) has demonstrated that in Western Australia we identify 8-10 new LS patients per year. Many of these patients did not report a family history of cancer and would not have been identified without this screening. Approximately $17 \%$ of CRC screened for MSI/IHC were identified as appropriate for referral for LS genetic testing. The incidence of germline mutations in these cases was approximately $25 \%$. Only a minority of these cases did not attend the WA Familial Cancer Clinic for various reasons to be discussed.

Based on our experience, three key elements required for successful population-based detection of LS in Australia will be discussed. We propose the adoption of a coordinated approach at a national level that includes the above key elements. This endeavour is a priority of the recently established Inherited Cancer Connect Partnership (ICCon), a group of clinicians and scientists focused on improving the outcomes of people with rare inherited cancer syndromes.

\section{AACG Oral 8 \\ MUTATIONS IN THE WNT SIGNALLING PATHWAY AND EYE DEVELOPMENT}

Ivan Prokudin', Yongjuan Chen', Rebecca Storen', John Grigg ${ }^{2}$, Robyn Jamieson ${ }^{1,2}$

${ }^{1}$ Children's Medical Research Institute, Sydney, NSW, Australia

${ }^{2}$ The Children's Hospital at Westmead and Save Sight Institute, University of Sydney, Sydney, NSW, Australia

Microphthalmia and coloboma are rare genetic conditions that may be associated with other ocular malformations including anophthalmia, anterior segment dysgenesis and cataracts. Disease genes include SOX2, OTX2, CHX10, BMP4 and RAX, but in many patients the disease gene is not known. In addition to the low mutation detection rate for patients with these conditions, there is often low penetrance and variable expression which may be due to the presence of genetic modifiers. WNT signalling is important in various developmental processes, including eye development. We sequenced a candidate gene in the WNT signalling pathway in a cohort of 180 patients with cataract/microcornea, coloboma/microphthalmia and Peters anomaly conditions, revealing 3 novel mutations in 5 probands. Additional next-generation sequencing in the 5 patients did not reveal any mutations in the known disease genes. The mu- tations were subjected to luciferase assay using the TOPFLASH reporter system. One mutation resulted in a significant difference in WNT activity. To further evaluate the candidate gene in early eye development we generated a mutant mouse line with variable WNT activity regulated by the dosage of the candidate gene. Subsequent examination of the morphology and markers of neuroretinal, retinal pigment epithelium (RPE) and lens development of the generated mouse mutants revealed regionalization of WNT signalling activity. We also noticed reduced expression of the neuroretinal marker Chx10 and ectopic expression of the RPE marker Mitf in the mutant mouse line. Our results demonstrate the key function of WNT signalling in precise spatial and temporal in early eye development.

\section{AACG Oral 9 \\ CLINICAL UTILITY OF THE MODIFIED MANCHESTER SCORE FOR BRCA GENE TESTING AT GENETIC SERVICES WESTERN AUSTRALIA \\ Anna Jarmolowicz' ${ }^{1}$ Helen Mountain ', Guicheng Zhang' ${ }^{2}$, Nicholas Pachter ${ }^{1,3}$ \\ ${ }^{1}$ Genetic Services of Western Australia, Perth, WA, Australia \\ ${ }^{2}$ School of Public Health, Curtin University, Perth, WA, Australia \\ ${ }^{3}$ Department of Paediatrics and Child Health, University of Western Australia, Perth, WA, Australia}

The purpose of the study is to evaluate the prediction tool we currently utilize, the Modified Manchester Score (MMS), to determine eligibility for BRCA testing at Genetic Services Western Australia, in light of increasing test requests and the question of exception cases. Between January 1, 2009 and December 31, 2012546 diagnostic BRCA tests were ordered. 389 cases (71.25\%) met MMS testing criteria cut-off $(\geq 15)$. The detection rate above this cut-off was $17 \%(66 / 389)$, with sensitivity $89 \%$ and specificity $31 \% .157$ (28.75\%) did not meet MMS criteria; however, they were deemed eligible for testing for various clinical reasons (exception to Manchester group). In this group the detection rate was 5.1\% (8/157). Common reasons for testing below the cut-off were: no contact with family, small family size, family history of breast and ovarian cancer, epithelial ovarian cancer and triple negative breast cancer pathology. Further analysis of exception cases showed that 13/65 cases of ovarian cancer and 27/92 cases of grade 3 triple negative breast cancer did not meet MMS testing criteria. 1 (7.7\%) and 2 (7.4\%) mutations respectively were found in these groups. Increasing the minimum MMS testing cut-off from $\geq 15$ to $\geq 16$, saw no decline in the sensitivity, but a slight improvement in specificity of finding a BRCA mutation. Few mutations were missed using the MMS. Testing exception cases may result in over testing, increased economic burden and psychosocial issues for patients without improved mutation detection rates.

\section{AACG Oral 10}

ISOLATED AUTISM IS NOT AN INDICATION FOR SLO TESTING

$\underline{\text { Peter Kaub }}^{1,2}$, Peter Sharp' , Janice Fletcher ${ }^{1,2}$

${ }^{l}$ Genetics \& Molecular Pathology, SA Pathology, Women's \& Children's Hospital, Adelaide, SA, Australia

${ }^{2}$ Molecular Pathology, SA Pathology, Royal Adelaide Hospital, Adelaide, SA, Australia

Smith-Lemli-Opitz (SLO) syndrome is an autosomal recessive disorder of cholesterol synthesis due to deficiency in 7dehydrocholesterol (7-DHC) reductase. SLO is associated with specific dysmorphology, microcephaly, syndactyly, cleft palate, growth retardation, genital ambiguity and intellectual disability with autistic features. The autistic features may respond to cholesterol supplementation. Increased levels of 7-DHC are a marker for SLO and 
routinely used for testing of autistic children. A total of 6 years of requests for 7-dehydrocholesterol (7-DHC) testing at SA Pathology were audited (2008-13). Data were broadly classified according to clinical indications, including isolated autism, developmental delay, seizure, dysmorphism, neurological, anatomic, genital or metabolic anomalies. Data were subclassified as single or multiple clinical indication/s or no clinical information supplied; 1,084 tests were performed, 15 cases of SLO were detected. Only 6 cases of SLO were from post-natal serum or plasma samples from living patients. Five of those cases had multiple clinical indications, with only one having a single indication. In plasma/serum mean 7-DHC level when autism was a single or multiple indication was only $0.8 \mu \mathrm{mol} / \mathrm{L}$, compared to $264.7 \mu \mathrm{mol} / \mathrm{L}(\mathrm{NR}<2.0)$ for confirmed SLO cases. No cases of SLO were diagnosed based on an isolated clinical indication of autism or where no clinical information was supplied. Experience of several years of 7-DHC testing at SA Pathology indicates that autism as an isolated clinical trait is not associated with SLO. Therefore, 7-DHC measurement should not be included in the testing panel when autism spectrum disorders are the only clinical indication.

Concurrent 7: Australasian Society of Genetic Counsellors Oral Presentations

\section{ASGC Oral 5 \\ 'VERY OFTEN THE ANSWER'S NOT BLACK OR WHITE': INVESTIGATING COMMUNICATION DURING PEDIATRIC CLINICAL GENETIC CONSULTATIONS.}

Jean Paul ${ }^{1,2}$, Sylvia Metcalfe ${ }^{1,2}$, Lesley Stirling', Jan Hodgson ${ }^{1,2}$

${ }^{1}$ The University of Melbourne, Melbourne, VIC, Australia

${ }_{2}^{2}$ Murdoch Children's Research Institute, Melbourne, VIC, Australia

Background: New genomic tests provide hope for a diagnosis for many children with developmental delay. However, results generated through use of these technologies increases the complexity of communication and uncertainty during genetic consultations. Little is known about the process of pediatric genetic consultations. Aim: We explored communicative processes coupled with parent and clinician experiences of consultations where children with developmental delay were referred to a genetics clinic. Methods: This qualitative project investigated consultations across four Australian states. Theoretical framework: Symbolic Interactionism - meaning is derived, created and modified through social interactions. Data: audio-recorded consultations $(n=32)$, parent pre-consultation surveys $(n=32)$, and post-consultation interviews with parents $(n=$ $32)$ and clinicians $(n=11)$. Content, thematic and discourse analyses were completed across the data sets. Results: While the content of consultation was similar, different communication 'styles' were apparent and variable approaches were used when explaining genetic results. Interviews with parents revealed that overwhelmingly, they appreciated and were reassured by the consultation. The vast majority reported a positive relationship with their clinician and felt the genetic information had been explained in a very useful manner. During interviews, clinicians reported many professional challenges working in this area, both practical and emotional, especially frustrations of being unable to answer parents' questions regarding the cause of the child's delay. Conclusion: Detailed analyses of three complementary data sources provided rigorous and unique perspectives on impacts regarding the introduction of new genetic technologies for clinicians and parents. Findings from this study will inform best practice in this area of medical communication.

\section{ASGC Oral 6 \\ IT WASN'T A DISASTER OR ANYTHING: PARENTS' EXPERIENCES OF THEIR CHILD'S UNCERTAIN CHROMOSOMAL MICROARRAY RESULT}

Ella Wilkins $^{1,2,3}$, Alison Archibald ${ }^{1,2,3}$, Margaret Sahhar ${ }^{1,2,3}$, Sue White ${ }^{1,2}$

${ }^{1}$ Department of Paediatrics, University of Melbourne, VIC, Australia

${ }^{2}$ Victorian Clinical Genetics Services, VIC, Australia

${ }^{3}$ Murdoch Children's Research Institute, VIC, Australia

The aim of this study was to understand parents' experiences of an uncertain chromosomal microarray (CMA) result for their child. This research utilized a qualitative approach with a phenomenological theoretical perspective. Families were ascertained via the Victorian Clinical Genetics Services and all families had a child with $16 \mathrm{p} 11.2$ or $15 \mathrm{q} 13.3$ microdeletion. Semi-structured interviews were conducted with nine parents of eight children aged between 2 and 11 years old at the time of testing. Interviews were transcribed verbatim and thematic analysis was used to identify themes within the data. Participants were unprepared for the CMA test and accompanying abnormal result. Despite a complex perception of the extent of their child's condition and a mixed understanding of the clinical relevance of the result, they were accepting of the limitations of current medical knowledge, and appeared to have readily adapted to the result. The test result was empowering for parents in terms of access to medical, educational and financial services; however, they articulated significant unmet support needs. Participants expressed hope for the future, in particular that more information would become available over time, and were open to the possibility of further testing for their child. This research has demonstrated that parents of children who have an uncertain CMA result appear to adapt to uncertainty and limited available information. Furthermore, parents valued honesty and empathic ongoing support from health professionals despite limited information. Genetic health professionals are well positioned to provide such support, aid families' adaptation to their situation and promote empowerment.

\section{ASGC Oral 7 \\ LIVING WITH UNCERTAINTY: THE ROLE OF HOPE, SOCIAL SUPPORT AND ILLNESS COGNITION IN RAISING A CHILD WITH AN UNDIAGNOSED MEDICAL CONDITION.}

Tatiane Yanes', Barbara Biesecker ${ }^{2}$, Aideen Mcinerney-Leo ${ }^{3}$, Linda Humphreys'

${ }^{1}$ School of Biomolecular \& Physical Sciences, Griffith University, Brisbane, QLD, Australia

${ }^{2}$ National Human Genome Research Institute, National Institutes of Health, Bethesda, $M D, U S A$

${ }^{3}$ Diamantina Institute, University of Queensland Diamantina Institute, Brisbane, QLD, Australia

This study aimed to explore the experiences of parents raising a child with an undiagnosed medical condition by: describing parental uncertainty; assessing the role of hope, social support and illness cognition in adaptation; and exploring parents' satisfaction with support services available. Previous studies have shown parents in this population have to cope and adapt to living with high levels of uncertainty. This uncertainty permeates many aspects of parents' lives and imposes challenges when making short and long-term plans. Data was collected through an online survey. Recruitment occurred via support groups and online parenting forums, and 51 responses were included in the analysis. Data analysis suggested that in this population parents appraised uncertainty as a threat. Uncertainty was not identified as having a significant association to any domains of adaptation. Multivariate analysis identified a number of significant associations between key variables and domains of adaptation, with perceived benefits of the child's illness having the strongest association. Parents believed there were significant gaps in the support 
received, with many believing additional support services would be available if their child was diagnosed. The majority of parents believed a diagnosis was of high importance, and this not associated with the length of time without a diagnosis. This study provided insight into the challenges parents face when raising a child with an undiagnosed medical condition. It is important for genetic health professionals to be aware of these factors as this will enhance their ability to support their clients and implement strategies to facilitate adaptation.

\section{ASGC Oral 8 \\ POPULATION GENETIC CARRIER SCREENING FOR CYSTIC FIBROSIS, FRAGILE X SYNDROME AND SPINAL MUSCULAR ATROPHY: EXPLORING EXPERIENCES OF CARRIERS IDENTIFIED THROUGH THE VCGS REPRODUCTIVE GENETIC CARRIER SCREENING PROGRAM \\ Catherine Beard $^{1,2}$, Louisa Di Pietro ${ }^{3,4}$, David Amor ${ }^{1,4}$, Alison Archibald ${ }^{1,4}$ \\ ${ }^{1}$ University of Melbourne, Melbourne, VIC, Australia \\ ${ }^{2}$ Murdoch Children's Research Institute, Melbourne, VIC, Australia \\ ${ }^{3}$ Genetic Support Network of Victoria, Melbourne, VIC, Australia \\ ${ }^{4}$ Victorian Clinical Genetics Services, Melbourne, VIC, Australia}

Due to advancing genetic technologies, carrier screening for multiple inherited conditions can now be offered within the population. This research aimed to explore how women experience undergoing carrier screening for three common inherited conditions: cystic fibrosis (CF), spinal muscular atrophy (SMA) and fragile $\mathrm{X}$ syndrome (FXS), through the Victorian Clinical Genetics Services (VCGS) Reproductive Genetic Carrier Screening (RGCS) program. Adopting a qualitative approach using phenomenology as the theoretical framework, the study utilized in-depth semi-structured interviews, which were transcribed verbatim. The transcripts were coded using thematic analysis to identify emerging themes. Eight female participants took part in this study: five received a carrier result for SMA and three for CF. The majority of participants were pregnant during screening and described the decision to have the test as straightforward. Participants experienced emotional responses such as anxiety and stress whilst waiting for their partner's test result and also completed online research to find out more about the relevant condition during this time. Participants supported population carrier screening, preferably offered prior to conception. The findings of this study confirmed that genetic counselors (GCs) play an essential role within this program by providing support to couples after they receive a carrier result, particularly given the varying consent processes undertaken prior to screening. The provision of suitable Internet resources and GC-facilitated guidance to access reliable information would empower couples and assist the coping process. Improving awareness of the availability of population carrier screening within the community will also help improve knowledge levels and facilitate preconception screening.

\section{ASGC Oral 9 \\ GENETIC COUNSELING CHALLENGES IN OSTEOGENESIS IMPERFECTA}

David Sillence', Rosie Fell', Alexandra Groves², Cheryl Cotton', Edwina Rickard

${ }^{1}$ Children's Hospital at Westmead, Sydney, NSW, Australia

${ }^{2}$ Hunter Regional Genetics, Waratah, NSW, Australia

The Osteogenesis Imperfecta syndromes, also known as 'Brittle Bone' group of genetic skeletal disorders, result from mutations in over 18 distinct genes loci. In 2010, the International Nosology Committee (INCDS) grouped the phenotypes arising from these mutations into 5 major groups (Osteogenesis Imperfecta types 1-5). With the exception of OI type 5, each group shows further het- erogeneity. For this reason the INCDS recommended the use of a phenotypic classification. The phenotypic heterogeneity reflects the effect of the type of mutation (nonsense, missense, splicing, deletion/insertion, frameshift), the position of the mutation in the translated polypeptide and the effect on folding and functional activity of the protein as well as its trafficking from the cell. There are still as yet unexplained mechanisms which result in wide expressivity. There is both allelic heterogeneity (mutations in the two genes COL1A1 and COL1A2 genes result in at least 4 phenotypes) and allelic heterogeneity (at least 18 distinct gene loci). The phenotypic consequences of mutations in 4 of these genes may segregate in an autosomal dominant mode and 2 of these genes in an X-linked recessive mode. We have developed counseling paradigms for these 5 phenotypic groups and used these to guide genetic counseling for these 'disorders'. We have used these paradigms to counsel new and former patients. There is a high acceptability for the concept of preimplantation genetic diagnosis PGD in dominantly inherited disorders among young people with OI.

\section{ASGC Oral 10 \\ BEGINNING A GENETIC COUNSELING SERVICE IN A PRIVATE OBSTETRIC ULTRASOUND PRACTICE}

\section{Alice Weeks}

Specialist Women's Ultrasound, Melbourne, VIC Australia

Genetic counseling employment has traditionally been based in public hospitals. There is, however, an increasing number of genetic counselors working in private settings. The changing face of genetic counseling practice includes the expansion of employment for genetic counselors, in particular, in private health care settings. Using case examples and clinical data, this presentation outlines the experience of setting up a successful prenatal genetic counseling service in a private obstetric ultrasound practice in Melbourne. Discussion of key professional issues and challenges includes the definition of a new role, supervision, certification, and the introduction of genetic counseling to external practitioners. The aim of this presentation is to challenge and encourage genetic counselors to seek alternative and new types of employment.

\section{Concurrent 8: Australasian Society for Inborn Errors of} Metabolism Oral Presentations

\section{ASIEM Oral 5/6 \\ TRANSITION OF METABOLIC PATIENTS: WHO LOOKS AFTER MY PRECIOUS PATIENT?}

\author{
Ute Spiekerkoetter \\ Department of Pediatric and Adolescent Medicine, University Children's Hospital, \\ Freiburg, Germany
}

\section{ASIEM Oral 7 \\ THE JOURNEY OF A CHURCHILL FELLOWSHIP: TO EXPLORE PRACTICAL MANAGEMENT STRATEGIES TO IMPROVE THE QUALITY OF LIFE OF AUSTRALIAN CHILDREN WITH GENETIC METABOLIC DISORDERS AND THEIR FAMILIES - UK, SPAIN, 2012-13}

\section{Anita Inwood}

Royal Children's Hospital, Brisbane, Australia

The Winston Churchill Trust, established in 1965, provides all Australians with a unique opportunity for study. Successful Fellows are provided generous financial support to travel overseas to 
conduct research/study that is not possible in Australia. My study project was a 6-week scholarship to explore the practical management strategies to improve the quality of life of Australian children with genetic metabolic disorders. This journey commenced at the Willink Metabolic Unit,Manchester Children's Hospital, (3 weeks) and The Royal Salford Hospital (1 week) before moving to London to visit Evelina Children's Hospital and Great Ormond Street Hospital (1 week) with the final week attending the International Congress of Inborn Errors of Metabolism in Barcelona. Key areas identified for consideration in our metabolic unit are: (1) A lifetime metabolic service, (2) a two-staged transition to adult services, (3) an effective model for enzyme replacement therapy in the home and (4) a model for delivery of respite and palliative care in the hospice setting. This experience increased my professional knowledge in these areas and resulted in a report to health administrators in how service delivery can be delivered by different models of care that may present both improvement and cost savings.

\section{ASIEM Oral 8 \\ OUTCOMES OF LIVER TRANSPLANTATION FOR GLYCOGEN STORAGE DISEASE TYPE 1B (GSD1B) - THE NEW ZEALAND EXPERIENCE}

Rhonda Akroyd', Helen M Evans ${ }^{2}$, Nicola Clark ${ }^{3}$, Callum Wilson'

${ }^{1}$ National Metabolic Service, Starship Children Health, Auckland City Hospital, Auckland, New Zealand

${ }^{2}$ Paediatric Hepatology and Gastroenterology, Starship Children Health, Auckland City Hospital, Auckland, New Zealand

${ }^{3}$ Nutrition Services, Christchurch Hospital, Christchurch, New Zealand

Background: GSD1b is associated with significant morbidity, namely hypoglycaemia, lactic acidosis, hepatomegaly, neutropenia, recurrent infections, mouth ulcers, arthritis, hyperuricaemia, hyperlipidaemia, and inflammatory bowel disease (IBD). Complications may include osteoporosis and hepatic tumors with potential for malignant transformation. Liver transplantation (LT) in GSD1b treats the underlying defect, many of the clinical and biochemical manifestations and the hepatic malignancy risk. Cases: Two patients with GSD1b aged sixteen years have undergone deceased donor orthotopic LT. Both had required GCSF for neutropenia and gastrostomy insertion for nutrition support. Case 1 had recurrent hypoglycaemia, intellectual disability, IBD with recurrent oesophageal strictures, requiring multiple dilatations and stenting, poor dentition, deafness and significant social issues. Case 2 had HLA B27 positive arthritis, IBD and recurrent mouth ulcers. Both cases required nutrition support using polymeric formula and uncooked cornstarch (UCCS) during the day, and overnight Case 1 UCCS boluses and Case 2 continuous polymeric formula with additional glucose polymer. Height and BMI were $<0.4$ th and 91 st centiles respectively for Case 1 and 0.4 th and 75 th centiles respectively for Case 2. Discussion: Both cases are doing well at 15 and 9 months post LT with improved quality of life and improved school attendance. GCSF requirement has decreased, both are enjoying eating and Case 1 is swallowing well without oesophageal structuring. Case 2 now has quiescent arthritis. IBD appears to have significantly improved in both cases. Case 1 has normalized BMI (50th centile). Thus LT is associated with excellent outcomes for patients with significant morbidity related to GSD1b.

\section{ASIEM Oral 9 \\ THE DEVELOPMENT OF A GUIDELINE AND EDUCATION PACKAGE FOR THE IMPLEMENTATION AND MANAGEMENT OF BH4 THERAPY FOR PATIENTS WITH PHENYLKETONURIA (PKU) IN AUSTRALASIA}

Aoife Elliott $^{1}$, Annabel Sweeney ${ }^{2}$, Barbara Dennison ${ }^{3}$, Rhonda Akroyd ${ }^{4}$, Christie Graham $^{3}$, Maureen Humphrey ${ }^{5}$, Anne Rae ${ }^{6}$, Mary Westbrook ${ }^{7}$, Avihu Boneh ${ }^{5}$

\footnotetext{
${ }^{1}$ Mater Children's Hospital, Brisbane, QLD, Australia

${ }^{2}$ Women's and Children's Health Network, Adelaide, SA, Australia

${ }^{3}$ The Children's Hospital at Westmead, Sydney, NSW, Australia

${ }^{4}$ Auckland City Hospital, Auckland, New Zealand

${ }^{5}$ Royal Children's Hospital, Melbourne, VIC, Australia

${ }^{6}$ King Edward Memorial Hospital for Women, Perth, WA, Australia

${ }^{7}$ Westmead Hospital, Sydney, NSW, Australia
}

Aim: Tetrahydrobiopterin (BH4) is a relatively new treatment option for patients with Phenylketonuria (PKU). Funding was obtained by ASIEM Dietitians to develop a guideline and education package for the implementation and management of BH4 therapy for PKU in Australasia. Process: (1) A guideline development group was formed; (2) project officer recruited; (3) guideline scope agreed; (4) stakeholders identified: patients/carers of people with PKU and health professionals involved in PKU management in Australasia; (5) clinically relevant questions formed; (6) extensive literature review completed and appraised; (7) international survey to 'BH4 experts' undertaken to capture practical day-to-day experience not reported in the literature; (8) specifically designed Delphi Survey for health professionals developed to formally address issues either inadequately addressed in the literature or had evidence that could lead to contradictory recommendations and to explore issues relevant to our population and clinical setting; (9) telephone survey for PKU patients/carers to ensure patient/carer needs considered; (10) interstate ethics approval gained. The Delphi Survey was sent to 54 health professionals including clinicians, dietitians, nurses, social workers and psychologists involved in Australasian PKU management. Two rounds were completed with consensus reached on any statement with $\geq 75 \%$ agreement. The telephone survey was completed with patients/carers in Queensland, Victoria and New South Wales with representation across patient groups to identify areas of concern and education requirements related to the introduction of BH4 therapy. Outcome: The results will be used to develop the guideline and education package for dissemination and implementation as appropriate across Australasia.

\section{ASIEM Oral 10 \\ DIETETIC CHALLENGES IN MANAGING CARNITINE-ACYLCARNITINE TRANSLOCASE (CACT)} DEFICIENCY

Susan Thompson ${ }^{1,2}$, Barbara Dennison ${ }^{1,2}$, Kaustuv Bhattacharya'

${ }^{1}$ Genetic Metabolic Disorders Service, The Children's Hospital at Westmead, Sydney, NSW, Australia

${ }^{2}$ Department of Nutrition and Dietetics, The Children's Hospital at Westmead, Sydney, NSW, Australia

A female infant was diagnosed with carnitine-acylcarnitine translocase (CACT) deficiency following hypoglycaemia, hyperammonia, severe cardiac dysfunction and seizures in the first week of life. She was discharged home on day 23 on a low fat diet (5\% total energy from long chain fat) with total energy intake of $160 \mathrm{kcals} / \mathrm{kg} / \mathrm{day}$ from modified fat formula and glucose polymer, 4-hourly feeds with no fasting, supplementary beta-hydroxybutyrate $(600 \mathrm{mg} / \mathrm{kg} / \mathrm{d})$ and carnitine. She is now 4 years old and developing normally, with growth on the 50th centile. When well she eats age-appropriate foods supplemented with MCT, relying on a bottle for a high daily oral intake of formula. Education on appropriate food choices has been well understood and a gastrostomy was sited at 9 months of age for use 
when oral intake is poor and overnight feeds. She has a history of recurrent viral infections, with each episode life threatening, some with hypoglycemia, some with bradycardia. Dietetic challenges centre around achieving her high energy needs $(2 \mathrm{x}$ requirement at $180-200 \mathrm{Kcals} / \mathrm{kg} /$ day, with no evidence of malabsorption) using less than $<8 \%$ LCT, when well and unwell whilst maintaining balanced eating habits. Other challenges include distance from primary metabolic team with acute local hospital management.

Concurrent 9: Australasian Society of Cytogeneticists Oral Presentations

\section{ASOC ORAL 5 \\ NON-INVASIVE PRENATAL TESTING (NIPT): EXPERIENCE OF THE FIRST 500 CASES AT QFG}

Nicole Martin, Peter Field

Queensland Fertility Group, Brisbane, QLD, Australia

NIPT was first commercialized in 2011, with screening offered for the detection of trisomy 21 . There are now 5 commercial companies offering NIPT and the range of trisomies screened has been expanded to include 13,18 and 21 , with some companies offering sex chromosome abnormalities as well as triploidy. Twin screening remains to be conclusively validated. NIPT uses the fragments of fetal placental DNA circulating in the maternal plasma, referred to as the fetal fraction (ff).ff shows inter-patient variation, but generally increases with gestational age. ff is affected by maternal BMI; with a greater maternal circulating volume, the lower the fetal fraction and the more difficult a diagnosis becomes, depending on the test methodology. There are 2 main methodologies employed in NIPT, a counting method, which does not distinguish between maternal and fetal fragments in the maternal plasma and a SNP method which can distinguish between maternal and fetal fragments; this latter methodology also includes a paternal SNP screen. Maternal age in the group screened ranged from 25 to 44 years. The overall ff detected ranged from $1.2 \%$ to $23.9 \%$. Results were obtained in a ff range of $3.3 \%$ to $23.9 \%$. The gestational age at screening ranged from 9 to 19 weeks. Recollection, due to low ff was within the range predicted by the testing company; a result was more likely to obtained at a lower ff if a paternal DNA swab was submitted. Very elevated BMI cases failed to yield a result after recollection.

\section{ASoC Oral 6 \\ GENOME WIDE SNP ARRAY IS A VALUABLE TOOL IN THE INVESTIGATION OF FETAL AUTOPSY CASES.}

S. Brown', J. Nicholl', Y. Khong ${ }^{2}$, N. Manton ${ }^{2}$, J. Lipsett ${ }^{2}$, L. Moore ${ }^{2}$, E. Haan ${ }^{3}$, C. Barnett ${ }^{3}$, S. Yu'

${ }^{1}$ Genetics and Molecular Pathology, SA Pathology at Women's and Children's Hospital, Adelaide, SA, Australia

2 Surgical Pathology, SA Pathology at Women's and Children's Hospital, Adelaide, SA, Australia

${ }^{3}$ Medical Genetics, SA Pathology at Women's and Children's Hospital, Adelaide, SA, Australia

Array technology has been used to routinely investigate fetal autopsy cases in our laboratory since March 2011. These autopsy cases involved fetuses that died in utero or fetuses terminated due to abnormal ultrasound findings. Array CGH investigation of autopsy cases showed the incidence of a causative copy number variant (CNV) was 4.7\% (4/85). Since July 2013, SNP Array has been used to investigate 89 autopsy cases. Eleven CNVs were detected with six determined to be causative (6.7\%).SNP array also detects regions of homozygosity (ROHs) which can guide future DNA sequenc- ing effort to diagnose autosomal recessive (AR) disease. Seventeen cases (19\%) were found to have at least one $\mathrm{ROH}$. Case 1 is a fetus suspected to have AR Fraser syndrome. The SNP array detected 25 ROHs (207Mb, 6.9\% of human genome). One ROH included the FRAS1 gene, one of the genes known to cause Fraser syndrome. Subsequent sequence analysis of the FRAS1 gene revealed a homozygous, pathogenic mutation 9525T $>$ A. Cases 2 and 3 were siblings with polycystic kidney disease with no mutation of the PKHD2 gene. SNP array identified multiple ROHs in each fetus with 3 shared ROHs. Cases 4 and 5 were siblings with short ribbed polydactyly ?type IV with multiple ROHs and 3 shared ROHs. NGS is currently being conducted to identify AR mutations within the shared regions of ROHs. SNP array is a valuable tool for investigating fetal autopsy cases as CNVs, ROHs, isodisomy and mosaicism can be identified in a single test.

ASoC Oral 7

CYTOGENETICS: BACK TO THE FUTURE!

Ling Sun, Lucy Gugasyan

Cytogenetics, Monash Pathology, Monash Health, Melbourne, VIC, Australia

Various cytogenetic techniques can be used in conjunction to diagnose a patient's karyotype. Here we describe how four different methods helped characterize the karyotype of a 66-year-old female patient presenting with secondary amenhorrhea and features of Turner syndrome. The initial investigation on G-banded metaphases showed an apparent terminal deletion of one $\mathrm{X}$ chromosome at band q22.1 in 50 cells. A molecular karyotype to further characterize this finding additionally showed a $0.15 \mathrm{Mb}$ copy number gain of 16q24.3. Fluorescent in situ hybridization (FISH) revealed the presence of an unbalanced $t(X ; 16)$ and finally, replication studies demonstrated inactivation of the abnormal X chromosome. Hence, the clinical presentation of Turner syndrome is consistent with this imbalance elucidated by utilizing four different cytogenetic techniques.

Concurrent 10: Molecular Genetics Society of Australasia Oral Presentations

\section{MGSA Oral 5 \\ TARGETED SEQUENCE CAPTURE AND MASSIVELY \\ PARALLEL SEQUENCING TO DETECT MUTATIONS ASSOCIATED WITH MALIGNANT HYPERTHERMIA (MH)}

Anja H. Schiemann, Chay Fhun Low, Kathryn M. Stowell

Institute of Fundamental Sciences, Massey University, Palmerston North, New Zealand

Malignant Hyperthermia (MH) is an autosomal dominant pharmacogenetic disorder that affects the skeletal muscle calcium release from the sarcoplasmic reticulum. $\mathrm{MH}$ is triggered in susceptible individuals through exposure to inhalational anaesthetics during general anesthesia and results in a hypermetabolic state characterized by hyperthermia, muscle rigidity, tachycardia, hypoxaemia and metabolic acidosis, which if untreated, can lead to death. During an $\mathrm{MH}$ episode, the myoplasmic calcium level increases rapidly due to increased flux of calcium from the sarcoplasmic reticulum to the cytosol. In approximately $70 \%$ of $\mathrm{MH}$-susceptible families mutations are found in RYRI (skeletal muscle calcium-release channel), in $1 \%$ of cases mutations are found in CACNA1S (alpha $1 \mathrm{~S}$ subunit of the voltage-dependent $\mathrm{L}$ type calcium channel). In $30 \%$ of $\mathrm{MH}$-susceptible families mutations have not yet been identified. We have used whole exome sequencing or DNA capture of a limited 
set of genes combined with Next Generation Sequencing to identify candidate mutations in New Zealand/Australian families. Variants with high allele frequency in the general population and synonymous variants were filtered out. High Resolution Melting analysis was used to determine segregation of the candidate mutations in the families. Lymphoblastoid cell lines were used to functionally characterize candidate $R Y R 1$ mutations.

\section{MGSA Oral 6 \\ TARGETED RESEQUENCING FOR EARLY INFANTILE EPILEPTIC ENCEPHALOPATHIES IN A CLINICAL DIAGNOSTIC SETTING USING MASSIVELY PARALLEL SEQUENCING}

Gladys Ho' , Elizabeth Farnsworth', Katherine Holman ', Bruce Bennetts ${ }^{\text {1,2 }}$, John Christodoulou',

${ }^{1}$ Western Sydney Genetics Program, The Children's Hospital at Westmead, Sydney, NSW, Australia

${ }^{2}$ Disciplines of Paediatrics and Child Health \& Genetic Medicine, University of Sydney, Sydney, NSW, Australia

Early-onset epileptic encephalopathies are severe disorders of cognitive, sensory and motor development. Genetic testing in this field is hampered by the highly heterogeneous nature of these disorders, with marked phenotypic variability, overlapping clinical features and the involvement of multiple genetic loci. Due to the high costs and long turn-around times, traditional methods of molecular diagnosis are usually limited to a small number of candidate genes. Massively parallel sequencing (MPS aka next generation sequencing) provides an ideal platform for improving the genetic diagnosis of these disorders, by allowing a large number of genes to be tested simultaneously at a relatively low cost. Three different targeting approaches (Illumina TruSeq Custom Amplicon, Agilent Haloplex Custom Target Enrichment and Illumina TruSight Exome) have been trialled to interrogate up to 53 genes associated with disorders of early-onset seizures, Rett syndrome, neuronal ceroid lipofuscinoses or other disorders with overlapping clinical features. All three methods successfully identified candidate disease-causing variants in our patient cohort, but coverage performance varied depending on the method of targeting. Pick-up rate for patients screened for epileptic encephalopathy genes is low, with the majority of positive findings made in patients whose specific diagnoses were identified by other strong pointers (e.g., enzyme assays or characteristic clinical presentation). Cascade testing of other family members was important in the interpretation of variants of uncertain significance. The introduction of MPS into diagnostic laboratories has greatly facilitated the genetic diagnosis in patients with epileptic encephalopathy by reducing the time and cost required for testing.

\section{MGSA Oral 7 \\ EXOME SEQUENCING IN MOTORNEURONE DISEASE/ SPINAL MUSCULAR ATROPHY: IMPROVED DIAGNOSTICS AND COST-EFFECTIVENESS}

Tony Roscioli ${ }^{1,2}$, Hooi Ling Teoh ${ }^{3}$, Michelle Farrar ${ }^{3,2}$, Hugo Sampaio ${ }^{3}$, David Hanna ${ }^{4}$, Deborah Nickerson ${ }^{4}$, Josh Smith ${ }^{4}$, Eric Lee ${ }^{5}$, Michael Buckley ${ }^{5}$, Matthew Kiernan ${ }^{6}$

${ }^{1}$ Department of Medical Genetics, Sydney Children's Hospital, Sydney, NSW, Australia ${ }^{2}$ School of Women's and Children's Health, University of New South Wales, Sydney, NSW, Australia

${ }^{3}$ Department of Neurology, Sydney Children's Hospital, Sydney, NSW, Australia

${ }^{4}$ University of Washington, Genome Sciences, Seattle, WA, USA

${ }^{5}$ SEALS Genetics Laboratory, Prince of Wales Hospital, Sydney, NSW, Australia

${ }^{6}$ Bushell Chair of Neurology, Central Clinical School, Sydney University, Sydney, NSW, Australia

Motorneurone disease and spinal muscular atrophy are significant neurogenetic disorders. It is likely that the majority of motor neuron diseases have a genetic basis; however, gene mutations have not been defined in most patients. To date, the sequential testing of single genes has been slow and expensive and genetic mutations in most affected people remain to be identified. The recent availability of Next Generation Sequencing (NGS) has improved genetic testing and gene identification. We discuss NGS genetic data combined with clinical and neurophysiological assessments to provide pathophysiological insights into the motor neuron diseases. A minimum of two exomes have been performed in each family on the Illumina platform in 20 people with patterns of inheritance consistent with autosomal dominant and recessive traits. The results have identified pathogenic mutations in approximately $50 \%$ of the cohort. A potential novel gene expressed in the motor end plate has been identified in one consanguineous family for which investigations are ongoing. An analysis of whether a genomic diagnostic approach to patients with motor neurone diseases is cost-effective and alters traditional diagnostic algorithms is also assessed.

\section{MGSA Oral 8}

FAMILIAL HEMATOPOIETIC MALIGNANCIES FOR THE IDENTIFICATION OF INITIATORS AND MARKERS OF DISEASE PREDISPOSITION AND PROGRESSION

Christopher Hahn', Andreas Schreiber ${ }^{2}$, Jinghua Feng ${ }^{2}$, Cecily Forsyth ${ }^{7}$, Agnes Wong $^{3}$, Richard D'Andrea ${ }^{3}$, Sarah Moore' ${ }^{1}$, Jim Wiley ${ }^{5}$, Marshall Horwitz ${ }^{6}$, Stephen Fuller ${ }^{5}$, Graeme Suthers ${ }^{4}$, Hamish Scott ${ }^{1}$

\footnotetext{
1 Department of Genetics and Molecular Pathology, Centre for Cancer Biology, SA Pathology, SA, Adelaide, Australia

${ }^{2}$ ACRF Cancer Genomics Facility, Centre for Cancer Biology, SA Pathology, Adelaide, SA, Australia

${ }^{3}$ Haematology, SA Pathology, Adelaide, SA, Australia

${ }^{4}$ SA Clinical Genetics Service, Genetics and Molecular Pathology, SA Pathology, Adelaide, SA, Australia

${ }^{5}$ Department of Medicine, Sydney Medical School Nepean, The University of Sydney, Sydney, NSW, Australia

${ }^{6}$ Department of Pathology, University of Washington School of Medicine, Seattle, WA, USA

7 Jarrett Street Specialist Centre, North Gosford, NSW, Australia
}

Familial cases of haematopoietic malignancy (HM) offer the opportunity to identify novel predisposition genes that likely also impact on the more common sporadic cases. They also offer affected families a tool for screening for predisposed individuals and possibilities for treatment if the identified genes or mutations are druggable. We previously discovered GATA2 as a myelodysplastic syndrome (MDS)/acute myeloid leukaemia (AML) predisposing gene which has led to the identification of $\sim 50$ families worldwide with GATA2related disease, and a recommended standard of care for carrier individuals who develop disease.

We have performed whole exome sequencing (WES) and/or AmpliSeq 29 gene MDS/AML panel sequencing on the Ion Proton on affected and unaffected members from 50 families predisposed to HM. To date, we have sequenced over 200 samples and have not only developed a pipeline for sequence generation, alignment and variant calling (including detection of low frequency variants to assess clonal expansion), but also for variant annotation to facilitate pathogenicity calling. We have used the power of this approach to identify new germline mutations, to follow the mutational progression of disease through therapy, remission and relapse, and to identify pathogenic initiator or driver mutations in individuals prior to the onset of symptoms. This helps catalogue markers for early diagnosis of disease onset, contributes to prognostic data, enables time for development of therapeutic approaches including searching for bone marrow donors, and adds to understanding the biology of genes and genetic interactions that are common in HMs and those that unique to specific HMs. 
Concurrent 11: Education Session

\section{Workshop \\ HOW TO WRITE RESEARCH ARTICLES FOR PUBLICATION \\ Margaret Cargill',2 \\ ${ }^{1}$ School of Agriculture, Food \& Wine, University of Adelaide, Adelaide, SA, Australia \\ ${ }^{2}$ SciWriting, Magill, SA, Australia (www.writeresearch.com.au)}

This 90-minute workshop aims to help conference presenters take the next steps in moving their conference presentation towards an article likely to get a respectful reading from the editors and referees of their target journal. It is based on the presenter's recent teaching text on this topic, and reflects 20 years' experience in helping researchers write science effectively. Participants should bring with them a paper copy of a research article relevant to their own field and published in a journal they would like to target - we will analyse aspects of these during the workshop. Topics to be addressed during the workshop include: (1) Developing article writing skills by analysing articles using applied linguistics frameworks; (2) Results as 'driver': developing and writing your 'take-home message' and selecting a target journal; (3) Article structures and referee criteria - the connections; (5) Introductions: 5 'stages' to a compelling justification.

Concurrent 12: Free Communications

\section{Oral 1 \\ LARS2 VARIATIONS ASSOCIATED WITH HYDROPS, LACTIC ACIDOSIS, SIDEROBLASTIC ANAEMIA AND MULTISYSTEM FAILURE \\ Lisa Riley $^{1,3}$, Joëlle Rudinger-Thirion ${ }^{5}$, Sandra Cooper ${ }^{2,3}$, John Christodoulou ${ }^{1,3,4}$ \\ ${ }^{1}$ Genetic Metabolic Disorders Research Unit, Kids Research Institute, Children's Hospital at Westmead, Sydney, NSW, Australia \\ ${ }^{2}$ Institute for Neuroscience and Muscle Research, Kids Research Institute, Children's Hospital at Westmead, Sydney, NSW, Australia \\ ${ }^{3}$ Discipline of Paediatrics \& Child Health, Sydney Medical School, University of Sydney, Sydney, NSW, Australia \\ ${ }^{4}$ Discipline of Genetic Medicine, Sydney Medical School, University of Sydney, Sydney, NSW, Australia \\ ${ }_{5}^{5}$ Architecture et Réactivité de l'ARN, Université de Strasbourg, CNRS, IBMC Strasbourg, France}

Mutations in LARS2 have previously been associated with Perrault syndrome (premature ovarian failure and hearing loss). LARS2 encodes mitochondrial leucyl-tRNA synthetase which is required for mitochondrial protein synthesis. In this study we report LARS2 variations associated with a severe multisystem disorder. The proband was born prematurely ( 27 weeks) with severe lactic acidosis, hydrops and sideroblastic anaemia. She had multi-system complications with hyaline membrane disease, impaired cardiac function, a coagulopathy, pulmonary hypertension, progressive renal disease, and succumbed at 5 days of age. Whole exome sequencing was undertaken to identify a genetic diagnosis, and functional studies performed to investigate pathogenesis. Whole exome sequencing of patient DNA revealed compound heterozygous mutations in LARS2 (c.1289C > T; p.Ala430Val and c. $1565 \mathrm{C}>\mathrm{A}$; p.Thr522Asn).
The c.1565C $>$ A (p.Thr522Asn) mutation has previously been associated with Perrault syndrome and both variants are predicted to be damaging. There was some overlap of clinical features of this patient with those reported for YARS2 mutations (sideroblastic anemia and lactic acidosis); however, unlike YARS2 patients, muscle samples did not display any clear mitochondrial RC enzyme deficiency. Western blotting of patient muscle, liver and fibroblasts did not reveal any deficiency in LARS2 or OXPHOS protein levels. Aminoacylation assays are being undertaken to determine the effect of each of these mutations on the catalytic efficiency of LARS2. We speculate that LARS2 mutations may result in variable phenotypes. Further specific functional studies will clarify whether the LARS2 variations identified were responsible for the severe multisystem clinical phenotype seen in this baby.

\section{Oral 2}

UQCC2 AND PET100 MUTATIONS CAUSE ASSEMBLY DEFECTS OF MITOCHONDRIAL OXIDATIVE PHOSPHORYLATION COMPLEXES III AND IV

\author{
David Thorburn ${ }^{1,3}$, Elena Tucker ${ }^{1,3}$, Sze Chern Lim ${ }^{1,3}$, Hayley Mountford ${ }^{1,3}$, Ann \\ Frazier $^{1,3}$, Alison Compton ${ }^{1,3}$, Katherine Smith ${ }^{4,3}$, Melanie Bahlo ${ }^{4,3}$, Damien \\ Bruno $^{1,2}$, Heidi Peters ${ }^{2}$, David Mowat ${ }^{5}$, Bridget Wilcken ${ }^{6,7}$, John \\ Christodoulou $^{6,7}$, Bas Wanschers ${ }^{8}$, Radek Szklarczyk ${ }^{8}$, Leo Nijtmans ${ }^{8}$, Martijn \\ Huynen ${ }^{8}$, Matthew McKenzie? ${ }^{9}$ David Stroud ${ }^{9}$, Michael Ryan? \\ ${ }^{1}$ Murdoch Childrens Research Institute, Melbourne, VIC, Australia \\ 2 Victorian Clinical Genetics Services, Melbourne, VIC, Australia \\ ${ }^{3}$ University of Melbourne, Melbourne, VIC, Australia \\ ${ }^{4}$ Walter and Eliza Hall Institute of Medical Research, Melbourne, VIC, Australia \\ ${ }^{5}$ Sydney Children's Hospital, Sydney, NSW, Australia \\ ${ }^{6}$ Children's Hospital at Westmead, Sydney, NSW, Australia \\ ${ }^{7}$ University of Sydney, Sydney, NSW, Australia \\ ${ }^{8}$ Radboud University Medical Centre, Nijmegen, The Netherlands \\ ${ }^{9}$ La Trobe University, Melbourne, VIC, Australia
}

The 5 enzyme complexes of mitochondrial oxidative phosphorylation (OXPHOS) comprize a total of more than 80 subunits but most patients with single enzyme defects lack mutations in these subunits. We used massively parallel sequencing (MPS) of patients to identify two novel OPXHOS assembly factors and proved causality via lentiviral correction studies in patient fibroblasts. First, we identified a homozygous splicing mutation in the C6orf125 gene (now renamed $U Q C C 2$ ) in a consanguineous Lebanese patient with complex III deficiency, severe intrauterine growth retardation, neonatal lactic acidosis and renal tubular dysfunction. Sequence-profile based orthology prediction shows UQCC2 is an ortholog of the Saccharomyces cerevisiae complex III assembly factor, Cbp6p, although its sequence has diverged substantially. UQCC2 interacts with UQCC1, the predicted ortholog of the Cbp6p binding partner Cbp3p, and both are required for synthesis \&/or assembly of the mtDNA-encoded cytochrome $b$. Second, we studied eight complex IV-deficient patients with Leigh syndrome from six families of Lebanese origin. Complementation analysis suggested they had mutation(s) in the same gene but targeted MPS of 1,034 genes encoding known mitochondrial proteins failed to identify a likely candidate. Targeted MPS of a linkage region on chromosome 19 identified a homozygous mutation (c.3G $>$ C, p.Met1?) in C19orf79 (now renamed PET100). We showed it is located in the mitochondrial inner membrane and forms a $\sim 300-k D a$ subcomplex with complex IV subunits. We estimated that the mutation arose at least 520 years ago, explaining how the families could have different religions and different geographic origins within Lebanon. 


\section{Oral 3}

QUALITY STANDARDS FOR DATABASES OF DNA

\section{SEQUENCE VARIANTS}

Bruce Bennetts' ${ }^{\text {, Melody Caramins }}{ }^{2}$, Debra Graves ${ }^{3}$, Arthur Hsu ${ }^{4}$, Chiyan Lau ${ }^{3}$, Scott Mead ${ }^{5}$, Cliff Meldrum ${ }^{6}$, Graeme Suthers ${ }^{7}$, Graham Taylor ${ }^{4}$, Vanessa Tyrrell $^{3}$

${ }^{1}$ The Western Sydney Genetics Program, The Children's Hospital Westmead, Sydney. NSW, Australia

2 SDS Pathology, North Ryde, NSW

${ }^{3}$ The Royal College of Pathologists of Australasia (RCPA), Surry Hills, NSW, Australia

${ }^{4}$ Department of Pathology, University of Melbourne, Melbourne, VIC, Australia

5 South Eastern Area Laboratory Services, Prince of Wales Hospital, Sydney, NSW, Australia

${ }^{6}$ Hunter Area Pathology Service, John Hunter Hospital, Newcastle, NSW, Australia

7 SA Clinical Genetics Service, SA Pathology, Adelaide, SA, Australia

It is now routine practice to compare sequence variations identified during clinical genetic testing with variants recorded in a wide range of databases as well as in the scientific literature. New technologies are producing an even greater demand for analysis and interpretation, forming a substantial proportion of the diagnostic workload. Although numerous mutation databases already exist, there are few that meet the accuracy and reproducibility required for clinical diagnostics. Current databases are of variable quality and may contain errors in variant calls, non-standardized nomenclature, incomplete pathogenicity associations and limited phenotypic information linked to genomic data. These all represent limitations and risks to the quality of patient care. No standards or equivalent mechanisms exist for the accreditation of databases to ensure the accuracy and quality of uploaded data into any central repository to meet the needs of the clinical diagnostics environment. The RCPA in collaboration with the HGSA and the Human Variome Project (HVP) is developing standards for DNA sequence variation databases intended for use in the clinical environment. The framework for the development of these standards has addressed the following key areas: purpose and scope, governance, establishment, protection privacy security, content and functionality, curation, sharing, and professional use, management and workforce training. The draft standards are discussed to demonstrate and encourage the development and management of clinical grade databases, and secure sharing of accurately and appropriately curated variants and associated phenotypes. These standards will accelerate the delivery of accurate, actionable, and efficient clinical reports to improve patient management and outcomes.

\section{Oral 4 \\ IN THE CONTEXT OF MULTIGENIC ANALYSES OF COMPLEX GENETIC DISORDERS, SHOULD THE PATHOGENICITY OF A VARIANT BE ASSESSED IN ISOLATION FOR A CLINICAL APPLICATION?}

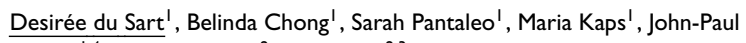
Plazzer $^{1,4}$, Ivan Macciocca ${ }^{2}$, Paul James ${ }^{2,3}$

${ }^{1}$ Molecular Genetics Laboratory, Victorian Clinical Genetics Services, Murdoch Children's Research Institute, Melbourne, VIC, Australia

${ }^{2}$ Victorian Clinical Genetics Services, Murdoch Children's Research Institute, Melbourne, VIC, Australia

${ }^{3}$ Clinical Genetics, Royal Melbourne Hospital, Melbourne, VIC, Australia

${ }^{4}$ InSIGHT Database Curator, Royal Melbourne Hospital, Melbourne, VIC, Australia

Inherited cardiac diseases are clinically important, genetically determined disorders, as well as significant causes of sudden death. Genetic testing for these conditions impacts on treatment protocols and clinical management options for the patient and at-risk family members. These conditions have a complex genetic pathology. They are often heterogeneous disorders with overlapping clinical symptoms, which may not always be penetrant. Determining whether variants are pathogenic, influence clinical severity or just normal variation within the genes is complex. We use a 101-gene, SureSelect capture NGS panel to screen for the arrhythmias (AP), cardiomyopathies (CP), aortopathies (ArP) and sudden death (SD) conditions. Data analysis is through a custom pipeline and the complete coding sequences of all genes are analyzed to a minimum coverage of $30 \mathrm{X}$ and quality score 15 . We report all variants with a $<1 \%$ frequency that have evidence of clinical impact. To date we have tested more than 400 proband samples, followed by segregation analyses in atrisk family members. Clinically actionable variants were identified in $71 \%, 81 \%, 68 \%, 80 \%$ of patients for AP, CP, ArP and SD respectively. The initial classification, a biological classification, is based on published data, in-silico and conservation analyses. Assessing the variant in the context of other gene variants identified, evidence of clinical impact and family segregation, facilitates a clinical classification specific for the family. The clinical classification has been different to the biological classification in a number of families, emphasizing the importance of this assessment in clinical applications.

\section{Oral 5 \\ HYPERMETHYLATION OF GADD45A IS CO-ASSOCIATED WITH IDH1, IDH2, AND TET2 MUTATIONS AND PREDICTS POOR OVERALL SURVIVAL AND CHEMORESISTANCE IN AML}

Michelle Perugini ${ }^{1,2,3,4,5}$, Saumya Samaraweera ${ }^{1,2,4,5}$, Anna L. Brown ${ }^{1,2,4,5}$, Chung H. Kok ${ }^{5,7}$, Diana G. Salerno ${ }^{1,4}$, Nik Cummings $^{8}$, Silke Danner ${ }^{3}$, Andrew H. Wei ${ }^{8}$, L. Bik To ${ }^{2,3}$, lan D. Lewis ${ }^{2,3,4}$, Richard D'Andrea $1,2,3,4,5$

${ }^{1}$ School of Pharmacy and Medical Sciences, University of South Australia, Adelaide, SA, Australia

${ }^{2}$ Division of Haematology, SA Pathology, Adelaide, SA, Australia

${ }^{3}$ Department of Haematology, Royal Adelaide Hospital, Adelaide, SA, Australia

${ }^{4}$ Centre for Cancer Biology, SA Pathology, Adelaide, SA, Australia

${ }_{5}$ Centre for Stem Cell Research, The University of Adelaide, Adelaide, SA, Australia

${ }^{6}$ School of Medicine, The University of Adelaide, Adelaide, SA, Australia

${ }^{7}$ South Australian Health and Medical Research Institute, Adelaide, SA, Australia

${ }^{8}$ Department of Clinical Haematology, The Alfred Hospital and Monash University, Melbourne, VIC, Australia

GADD $45 A$ is a tumor suppressor gene that plays cell-type dependent roles in cellular stress co-ordinating DNA repair and de-methylation, cell cycle arrest, and pro-apoptotic or pro-survival responses. Methylation of four discrete $\mathrm{CpG}$ (CpG1-4) residues in the distal promoter of GADD45A is a hallmark of many solid tumors and has been associated with impaired cell stress signaling and reduced drug response. In AML, GADD45A silencing is widespread but poorly characterized. We have shown that hypermethylation of the distal promoter of GADD45A (CpG1-4) is a common event in AML, occuring in 93 of $222(42 \%)$ patients. GADD45A hypermethylation is associated with poor survival in AML overall (median OS: 10 vs $25 \mathrm{mths} ; p=.03$ ) and in the intermediate group of patients (median OS: 11 vs 33mths; $p=.04$ ). The association of $G A D D 45 A$ hypermethylation with poor overall survival was also validated in an independent patient cohort. Subsequent exome capture and Sequenom-based mutation screening has revealed a striking overlap between $G A D D 45 A$ hypermethylation and $I D H 1 / 2$ and TET2 mutations $(78 \% ; p<.0001)$. This co-association suggests that GADD45A may be a key functional target of the DNA demethylation pathway governed by $I D H 1 / 2$ and TET2. Consistent with this, we show in patients and AML cell lines that re-activation of GADD45A can be achieved through treatment with mutant IDH inhibitors and the hypomethylating agents including Decitabine, resulting in increased chemosensitivity to Daunorubicin. In summary, GADD45A promoter hypermethylation is a novel functional biomarker of outcome in AML and its silencing is critical to chemoresistance. 
Oral 6

MITOCHONDRIAL CARBONIC ANHYDRASE VA DEFICIENCY: A NEW INBORN ERROR OF METABOLISM CAUSING HYPERAMMONEMIA IN A NEONATE

James Pitt', Joy Yaplito-Lee', Sylvia Stockler-lpsiroglu ${ }^{2}$, William Sly ${ }^{3}$, Wyeth Wasserman $^{2}$, Clara van Karnebeek ${ }^{2}$

${ }^{1}$ Victorian Clinical Genetics Services, Murdoch Children's Research Institute, Melbourne, VIC, Australia

${ }^{2}$ Division of Biochemical Diseases, BC Children's Hospital, Vancouver, BC, Canada ${ }^{3}$ Department of Biochemistry and Molecular Biology, St. Louis University School of Medicine, St. Louis, MO, USA

Hyperammonemia in children is frequently caused by urea cycle disorders or organic acidurias. We recently identified mitochondrial carbonic anhydrase VA (CAVA) deficiency, due to mutations in the $C A 5 A$ gene, as another cause of hyperammonemia in early childhood. CAVA is one of several carbonic anhydrase enzymes catalyzing the conversion of carbon dioxide to bicarbonate for subsequent use in bicarbonate-requiring enzymes such as carbamoylphosphate synthetase 1 (CPS1), pyruvate carboxylase, propionyl-CoA carboxylase and 3-methylcrotonyl-CoA carboxylase. We illustrate this new disorder with the case of a male who presented on day 4 of life with lethargy, weight loss, jaundice and tachypnea. Ammonia was $422 \mu \mathrm{mol} / \mathrm{L}(<50)$ with normal urine orotic acid, features suggestive of CPS1 or NAGS deficiency. However, lactate was increased at $8.1 \mathrm{mmol} / \mathrm{L}$ and urine organic acid screening showed increases in ketones, lactate, 3-hydroxypropionic, 3-hydroxyisovaleric and 3-methylcrotonylglycine. Treatment with carglumic acid normalized ammonia levels and other biochemical abnormalities within 48 hours. The patient has remained well up to last contact at 11 months with normal metabolite levels. He was homozygous for a $C A 5 A$ splice site mutation (c.555G $>$ A) leading to skipping of exon 4. While the metabolite levels are not as pronounced as those seen in multiple carboxylase deficiencies, CAVA deficiency can be recognized by careful interpretation of biochemical profiles provided samples are collected during hyperammonemic episodes. It responds well to treatment with a good prognosis and should be considered as part of the differential diagnosis of hyperammonemias.

Concurrent 13: Free Communications

\section{Oral 7 \\ NEXT GENERATION SEQUENCING IN AN AUSTRALIAN COHORT WITH CONGENITAL CATARACTS AND MICROCORNEA}

Alan Ma ${ }^{1,7}$, Bruce Bennetts ${ }^{6,7}$, Gladys $\mathrm{Ho}^{6}$, Maree Flaherty ${ }^{1,5}$, John Grigg ${ }^{1,3}$, David Mowat ${ }^{4}$, Ivan Prokudin ${ }^{1,2}$, Robyn Jamieson ${ }^{1,7}$

${ }^{1}$ Eye and Developmental Genetics Research Group, Western Sydney Genetics Program, The Children's Hospital at Westmead, Sydney, NSW, Australia,

${ }^{2}$ Children's Medical Research Institute, Westmead, Sydney, NSW, Australia

${ }^{3}$ Save Sight Institute, Sydney, NSW, Australia

${ }^{4}$ Department of Clinical Genetics, Sydney Children's Hospital Randwick, Sydney, NSW, Australia

${ }^{5}$ Department of Ophthalmology, Children's Hospital Westmead, Sydney, NSW, Australia

${ }^{6}$ Department of Molecular Genetics, Children's Hospital Westmead, Sydney, NSW, Australia

${ }^{7}$ Discipline of Paediatrics and Child Health, Sydney Medical School, University of Sydney, Sydney, NSW, Australia

Congenital cataracts have a significant impact on visual acuity and are responsible for $10 \%$ of childhood blindness worldwide. Congenital cataracts can also be associated with other ocular abnormalities such as glaucoma, microphthalmia and anterior segment dysgenesis, as well as genetic and metabolic syndromes. Cataract-microcornea is a genetically heterogeneous condition with over 30 known genes responsible. This study applied a targeted next-generation sequencing approach to an Australian cohort of 40 probands with cataracts $+/$ - microcornea, targeting 32 cataract-associated genes. Both the Illumina Truseq custom amplicon and Illumina Trusight Clinical Exome approaches were used. This study demonstrates the power of targeted next generation sequencing for an accurate diagnosis, and its effect on management and prognosis. 31 variants of interest were found in $29 / 40$ (72\%) of families, 21 of these were novel variants. Further segregation analysis confirmed likely mutations in $23 / 40$ families $(57.5 \%)$, in 13 genes. Half the mutations were found in the cataract-associated crystallin genes. In 2 families, mutations were found in the $\mathrm{X}$-linked syndromal gene $N H S$, which refined the diagnosis and had important intellectual development and family planning implications. Mutations were also found in the aniridia-associated gene PAX6 in 2 families, broadening the phenotypic features of this gene and altering the patient's management and monitoring. This study also adds to our knowledge and understanding of mutations in rarely reported lens-associated genes such as MIP, VIM and MAF. Our study demonstrates the many benefits of targeted next generation sequencing, which we will continue to explore in other developmental eye disorders and retinopathies.

\section{Oral 8}

USING TRUSIGHT PANELS TO CREATE DISEASE-SPECIFIC GENE PANELS FOR MASSIVELY PARALLEL SEQUENCING TESTING

Gladys Ho' , Katherine Holman', Alan $\mathrm{Ma}^{2,3}$, Elizabeth Farnsworth', Robyn Jamieson $^{2,3}$, Bruce Bennetts ${ }^{1,2}$, John Christodoulou'

${ }^{1}$ Western Sydney Genetics Program, The Children's Hospital at Westmead, Sydney, NSW, Australia

${ }^{2}$ Disciplines of Paediatrics and Child Health \& Genetic Medicine, University of Sydney, Sydney, NSW, Australia

${ }^{3}$ Eye Genetics Research Group, Children's Medical Research Institute, Sydney, NSW, Australia

Massively parallel sequencing (MPS, aka next generation sequencing) is a powerful tool for diagnostic genetic medicine, decreasing the time and cost required for testing a large number of genes associated with overlapping phenotypes. For Mendelian disorders with a limited number of causative genes, disease-targeted gene panels may have greater clinical utility over whole exome or whole genome sequencing, by allowing greater depth of sequencing coverage and reducing the complexity of variant interpretation. On the other hand, the design and validation of a panel for every disorder of interest are both cost and time-prohibitive, especially when discovery of new candidate genes entails constant revision of the panel with each addition. Using the Illumina TruSight panels (Inherited Disorders, Exome and One), we defined a variety of disease panels focusing on genes with known clinical phenotypes. Sequencing at average read depth of 300x provided over $97 \%$ coverage over the entire panel. Point mutations and medium sized insertions and deletions (10-50 bp) were identified, providing genotype diagnoses in patients with an aortopathy, epileptic encephalopathy, developmental eye disorders, mitochondrial disorders, renal disorders, osteogenesis imperfecta, Fanconi anemia, autoinflammatory disorders and various metabolic disorders. TruSight panels represent a versatile option for screening genes associated with a wide range of different clinical phenotypes with the use of a single test method. Not only does this reduce the time and expense of testing, it allows for greater flexibility in reviewing subpanels of genes, maximizing coverage over genes of interest, and minimizing incidental findings. 


\section{Oral 9}

WHOLE EXOME SEQUENCING IN GENETIC CONDITIONS CAUSED BY LARGE AND/OR MULTIPLE GENES

Aideen Mclnerney-Leo' ', Mhairi Marshall', Paul Leo', Jessica Harris', Brooke Gardiner', Matthew A. Brown', Emma L. Duncan ${ }^{2,1}$

${ }^{1}$ University of Queensland Diamantina Institute, Brisbane, QLD, Australia

${ }^{2}$ Royal Brisbane and Women's Hospital, Brisbane, QLD, Australia

Whole exome sequencing (WES) is not commonly used for determining previously identified genetic diagnoses as most laboratories favor targeted capture of candidate genes. However, such approaches cannot easily incorporate new gene discoveries. We evaluated the performance of 'off-the-shelf' WES capture for individuals with Marfan syndrome (MFS) and autosomal dominant osteogenesis imperfecta $(\mathrm{OI})$ - caused by a small number of large genes; phaeochromocytomas (PCC) and paragangliomas (PGL) - caused by numerous genes; and short rib polydactyly syndromes (SRP), associated with large and numerous genes. WES was performed using Illumina TruSeq and Roche NimbleGen capture platforms and Illumina HiSeq2000 sequencing technology. The MFS/OI/PCC/PGL samples had been tested previously and WES results were assessed blindly and compared post analysis. WES identified previously reported mutations in 13/13 OI cases; 9/10 MFS cases and 11/11 PCC/PGL patients. In the SRP group recessive mutations were detected in known genes in 11 of 13 cases. One further case had a single deleterious mutation in WDR60 but a second mutation was not identified. A new candidate gene was identified in the final case. WES proved to be a sensitive, efficient, and rapid means of sequencing multiple genes; however, successful mutation identification requires careful consideration of platform selection and a variety of bioinformatics approaches.

\section{Oral 10 \\ NEUROGENETIC DISEASE DIAGNOSTICS BY TARGETED CAPTURE AND NEXT GENERATION SEQUENCING}

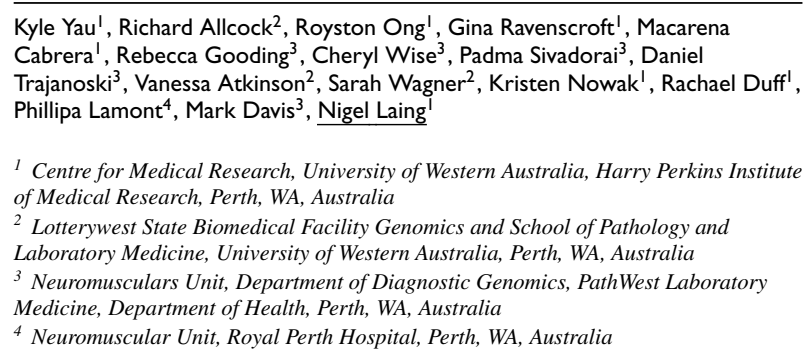

${ }^{1}$ Centre for Medical Research, University of Western Australia, Harry Perkins Institute of Medical Research, Perth, WA, Australia

${ }^{2}$ Lotterywest State Biomedical Facility Genomics and School of Pathology and Laboratory Medicine, University of Western Australia, Perth, WA, Australia

${ }^{3}$ Neuromusculars Unit, Department of Diagnostic Genomics, PathWest Laboratory Medicine, Department of Health, Perth, WA, Australia

${ }^{4}$ Neuromuscular Unit, Royal Perth Hospital, Perth, WA, Australia

Two problems for molecular diagnosis of neurogenetic disorders are high levels of genetic heterogeneity and involvement of large genes; for example, titin. These problems result in many patients not being diagnosed by current Sanger-sequencing based diagnostics. To offset these problems we have developed a TargetSeq (Life Technologies) capture panel consisting of 335 disease genes with mutations detectable by next generation sequencing (NGS). The genes were 276 neurogenetic and 59 cardiomyopathy genes, targeted together because of clinical overlap. The 335 panel genes were captured from pools of 16 bar-coded patient DNA samples and sequenced 16 patients at a time using Ion Proton ${ }^{\mathrm{TM}}$ (Life Technologies) sequencing. Variant analysis was by either custom Annovar or Cartagenia (Cartagenia, Inc.) based pipelines. Testing the panel identified $88 \%$ of the known small-scale mutations in 28 positive controls. Known mutations deliberately chosen in regions not se- quenced well by NGS were not detected. Using the FishingCNV package, we identified $90 \%$ of CNV controls, including $100 \%$ of CMT1A/HNPP duplication/deletion controls. Although one single exon $\mathrm{CNV}$ was detected, not all small CNVs were detected. We have screened $>300$ molecularly undiagnosed probands, identifying mutations in 59 disease genes, many of which were not previously analysed by the diagnostic laboratory due to Sanger sequencing costs. Using the targeted panel is not ethically different to traditional sequential Sanger sequencing diagnostics. The panel needs updating regularly with new disease gene discoveries. We now receive samples from all round Australasia for analysis of the 335-gene panel, which is cheaper than Sanger-based analysis of one medium-sized gene.

\section{Oral 11}

HCFC1 GAIN- AND LOSS-OF-FUNCTION MUTATIONS CAUSE NEURODEVELOPMENTAL DISORDERS, AND AFFECT NEURAL PROGENITOR CELL BEHAVIOR AND NEURONAL GROWTH.

\author{
Lachlan Jolly ${ }^{1,3}$, Lam Son Nguyen ${ }^{2}$, Jozef Gecz ${ }^{1,3}$ \\ ${ }^{1}$ Robinson Institute, University of Adelaide, Adelaide, SA, Australia \\ ${ }^{2}$ Foundation Imagine, Hopital Necker-Efants Malades, Paris, France, \\ ${ }^{3}$ School of Paediatrics and Reproductive Health, University of Adelaide, Adelaide, SA, \\ Australia
}

We recently discovered a non-coding regulatory change in $H C F C 1$ that associated with X-linked intellectual disability (XLID) in a large multigenerational family (MRX3). The single base change abolished binding of the YY1 transcription factor at a highly conserved sequence in the 5'UTR of $\mathrm{HCFCl}$, resulting in loss of $\mathrm{HCFCl}$ transcriptional repression. We employed ex-vivo models of embryonic neural development to show that this change was likely pathogenic; overexpression of $\mathrm{HCFCl}$ caused cell cycle exit of neural progenitor cells (NPCs), and reductions in neurite growth of hippocampal neurons. Intriguingly, loss-of-function $\mathrm{HCFCl}$ mutations have now also been reported to cause severe neurodevelopmental delay. We have identified four additional non-recurrent missense variants in HCFCl that segregate (where evidence is available) with ID in four families, and sought functional validation of pathogenicity. HCFC1 is a modular protein that harbors transcriptional activation and repressive activities, and mutations may manifest as either gain or loss of function. We investigated the effects of both loss- and mutation-of $H C F C 1$ function on the behavior of neural cells using ex-vivo assays. In contrast to over-expression, reduced $H c f c 1$ expression promoted the cell cycling of NPCs at the expense of differentiation, and enhanced neurite growth of neurons. All four variants were tested for their ability to affect neurite growth when overexpressed in wildtype neurons, and in neurons depleted of endogenous $H c f c 1$. Our work supplies information on the pathogenicity of $H C F C l$ variants found in patients with ID, and identifies relevant disease mechanisms that converge on cells present during embryonic stages of brain development. 
Oral 12

JEUNE SYNDROME: IDENTIFICATION OF A NEW CILIOPATHY GENE WITH EXOME SEQUENCING AND CASE PRESENTATION HIGHLIGHTING THE RELEVANT CLINICAL FEATURES.

Fiona McKenzie ${ }^{1,2}$, Nicholas Smith ${ }^{3,4}$, Aideen Mclnerney-Leo ${ }^{5}$, Paul Leo $^{5}$, Brooke Gardiner $^{5}$, Andreas Zank ${ }^{6,7}$, Matthew Brown ${ }^{5}$, Emma Duncan ${ }^{5,8,9}$

${ }^{1}$ Genetic Services of Western Australia, Perth, WA, Australia

${ }^{2}$ The University of Western Australia, School of Paediatrics and Child Health, Perth, WA, Australia

${ }^{3}$ Department of Histopathology, PathWest, Princess Margaret Hospital for Children and King Edward Memorial Hospital for Women, Perth, WA, Australia

${ }^{4}$ School of Pathology and Laboratory Medicine Faculty of Medicine, Dentistry and Health Sciences, University of Western Australia, Perth, WA, Australia

${ }^{5}$ The University of Queensland Diamantina Institute, Translational Research Institute, Brisbane, QLD, Australia

${ }^{6}$ Discipline of Genetic Medicine, The University of Sydney, Sydney, NSW, Australia

${ }^{7}$ Academic Department of Medical Genetics, Sydney Children's Hospital Network (Westmead), Sydney, NSW, Australia

${ }^{8}$ Department of Endocrinology, James Mayne Building, Royal Brisbane and Women's Hospital, Brisbane, QLD, Australia

${ }^{9}$ The University of Queensland, University of Queensland Centre for Clinical Research, Brisbane, QLD, Australia

We present the case of a 16-week fetus with multiple congenital anomalies, including intrauterine growth restriction, a turricephalylike skull shape, paramedian cleft of the upper lip extending into the palate, hypoplasia of the nasal bridge and nose, four limb postaxial hexadactyly, hydrocephalus, brain heterotopia, ventriculoseptal defect (VSD), adrenal hypoplasia, focally prominent hepatic ductal plates and early renal cystic dysplastic change. Skeletal changes included shortened and curved long bones, short ribs, mild platyspondyly and spur-like projections of the acetabular roof. The combination of features suggested a skeletal ciliopathy like Jeune syndrome. Whole exome sequencing on fetal DNA identified compound heterozygous mutations in IFT172, encoding the IFT-B component of the intraflagellar transport complex. Simultaneously, other groups also identified mutations in IFT172 in similar cases, resulting in its identification as a new ciliopthy gene. An increasing number of skeletal ciliopathy genes are being identified, many acting in an autosomal recessive/compound heterozygous fashion with significant implications for future children in affected families. This case is presented to highlight the clinical and radiological signs that are most suggestive of a diagnosis in this spectrum and assist in directing appropriate genetic testing. It also, along with other recent breakthroughs in Jeune syndrome, highlights that exome sequencing may prove to be the most cost-effective and efficient means of determining a diagnosis.

\section{Oral 13 \\ SUBMICROSCOPIC DUPLICATIONS AT Xp11.2 CONTRIBUTE TO INTELLECTUAL DISABILITY}

Ching Moey', Susan Hinze', Louise Brueton ${ }^{2}$, Benjamin Kaimen ${ }^{3}$, Chrisptopher Barnett ${ }^{4}$, Jillian Nicholl ${ }^{5}$, Jozef Gecz' ${ }^{1}$ Cheryl Shoubridge

\footnotetext{
${ }^{1}$ Robinson Institute, University of Adelaide, Adelaide, SA, Australia

${ }^{2}$ Clinical Genetics Unit, Birmingham Women's Hospital, UK

${ }^{3}$ Hunter Genetics, Newcastle, NSW, Australia

${ }^{4}$ South Australian Clinical Genetics Service, Adelaide, SA, Australia

${ }^{5}$ SA Pathology, Adelaide, SA, Australia
}

Copy number variations (CNVs) are a common cause of intellectual disability (ID). Determining the pathogenicity of CNVs remains challenging. Here we report three males with ID with submicroscopic duplications at Xp11.2. Using the Affymetrix Cytoscan HD array we have established the extent of the duplicated regions, encompassing two known ID genes IQSEC2 and KDM5C. No other known pathogenic CNVs were identified in these patients. Patient 1 presented with mild learning difficulties and autistic features. He has two affected maternal cousins. The $400 \mathrm{~kb}$ duplication identified disrupts the longest isoform of IQSEC2 and extending through to FAM156A. Patient 2 had global developmental delay, severe expressive speech delay and a maternally inherited $579 \mathrm{~kb}$ duplication that extends proximal of IQSEC2 and distal of SSX2. Patient-3 is $11 \mathrm{yrs}$ old, with severe ID and no words. He is not ambulant and has dysmorphic features. His $1,441 \mathrm{~kb}$ duplication involves, in addition to the region of case 1 and 2 also HUWE1 (known ID gene) and extends to a region distal of $X A G E 1 D$. Clinically this patient is more severe than other reported SMC1A and HUWE1 patients. The duplication arose de novo. Lymphoblastic cell lines from patient-3 showed significantly elevated levels of $S M C 1 A$ and $K D M 5 C$ transcripts, while patient 2 had only KDM5C mRNA elevated, consistent with the extent of their CNVs. Our data suggests that submicroscopic duplications at Xp11.2 containing known ID genes IQSEC2 and KDM5C might cause ID; however. it remains to be established which one (or both) are dosage sensitive in males. Interestingly, both genes escape $\mathrm{X}$-inactivation.

\section{Oral 14 \\ GATA2 GERMLINE AND SOMATIC MUTATIONS: AN INSIGHT INTO THE MOLECULAR PATHOGENESIS OF HAEMATOLOGICAL MALIGNANCIES.}

Chan-Eng Chong ', Parvathy Venugopal ${ }^{1,2}$, Manuela Klingler-Hoffmann ${ }^{2}$, Philippa Stokes $^{3}$, Young Lee' , Peter Brautigan' ', Grant Engler ${ }^{4}$, Jacqui Matthews ${ }^{3}$, Steven Lane $^{5}$, Anna Brown ${ }^{4,6}$, Richard D'Andrea ${ }^{4,6}$, Christopher Hahn ${ }^{1,7}$, Hamish Scott ${ }^{1,6}$

${ }^{1}$ Department of Genetics and Molecular Pathology, Centre for Cancer Biology, SA Pathology, Adelaide, Australia

${ }^{2}$ School of Molecular and Biomedical Science, University of Adelaide, Adelaide, SA, Australia

${ }^{3}$ School of Molecular Bioscience, The University of Sydney, Sydney, NSW, Australia

${ }^{4}$ Division of Haematology, Centre for Cancer Biology, SA Pathology, Adelaide, SA, Australia

${ }_{5}^{5}$ Queensland Institute of Medical Research, University of Queensland, Brisbane, QLD, Australia

${ }^{6}$ School of Pharmacy and Medical Sciences, Division of Health Sciences, University of South Australia, Adelaide, SA, Australia

${ }^{7}$ School of Medicine, University of Adelaide, Adelaide, SA, Australia

GATA2 is a zinc finger (ZF) transcription factor essential for the survival and proliferation of haematopoietic stem/progenitor cells. We first discovered heterozygous GATA2 germline $(g)$ mutations (lossof-functions (LOF) mutations) predisposing families to myelodysplastic syndrome/acute myeloid leukaemia (MDS/AML). Subsequently, additional heterozygous GATA2 germline mutations have been detected in patients with immunodeficiency and/or Emberger syndrome. Somatic $(s)$ heterozygous GATA2 have also been found in specific haematological malignancies. We and others demonstrate that whole gene deletion, as well as cis-regulatory mutations can result in similar phenotypes, suggesting haploinsufficiency as important in the development of GATA2-mutant AML. To decipher the disease mechanism, an allelic series of mutations in ZF2 of GATA2 representing the major disease phenotypes: MDS/AML ( $g$ T354M, $g$ T355del), AML-M5 and biallellic CEBPA AML ( $s$ R362Q) and MonoMac syndrome (immunodeficiency with MDS/AML, $g$ R398W) have been generated. All mutants appropriately target the nucleus and display reduced transcriptional activity. DNA binding affinity of GATA2 mutants was significantly attenuated when assessed using whole protein or purified ZF2 domain. Co-immunoprecipitation experiments demonstrated variable binding of GATA2 mutants to known binding partners. In clonogenic assays, haematopoietic stem cells expressing GATA2 mutants resulted in mutation-specific changes to cell growth and differentiation with a general shift from monocyte to granulocyte production. Consistent with LOF characteristics of mutant GATA2 in AML, we have identified a novel subpopulation of AML that express low GATA2, raising the possibility that this might be a more prevalent 
mechanism of generating cellular conditions conducive to progression or maintenance of the AML phenotype.

Concurrent 15: Free Communications

\section{Oral 15 \\ ETHICS AND GENOMIC MEDICINE: TO FEAR OR TO FRIEND?}

\section{Ainsley Newson}

University of Sydney, Sydney, NSW, Australia

This study describes and critiques the ethical issues arising in genomic medicine across the lifespan. However, before delving too far into the finer points of the ethical issues, I will first examine what exactly is novel about genomic medicine from an ethical perspective. Why, for example, can't we simply reapply existing ethical concepts and theories? Drawing on features such as scale and pervasiveness of genomic information, I will demonstrate why current ethical concepts are either over-stretched or of no use at all. Then, using relevant case studies from across the human lifespan, the ethical issues arising in genomic medicine will be described and analyzed. Particular attention will be paid to the concept of consent and its limitations in genomic medicine. Additionally, the dogma of genetic exceptionalism will be challenged. Finally, suggestions will be made for new ethical concepts that might be of particular use in genomic medicine. These will include the concept of family wellbeing and personal responsibility for health. With genomics in its infancy in Australasian health care, the timing is ideal for an ethical analysis that takes account of real-life clinical and research contexts. Ethical analysis of emerging techniques of genomics and their application will help ensure this technology is best used to benefit all of us. Results from this work will be relevant to researchers, clinicians and policy-makers working in Australasian genomic medicine.

\section{Oral 16 \\ A SHORT TIME, BUT A LOVELY LITTLE SHORT TIME: BEREAVED PARENTS' EXPERIENCES OF HAVING A CHILD WITH SPINAL MUSCULAR ATROPHY TYPE 1}

Emily Higgs' ${ }^{1}$, Belinda McClaren ${ }^{2}$, Margaret Sahhar ${ }^{1,3}$, Monique Ryan ${ }^{4}$

${ }^{I}$ University of Melbourne, Melbourne, VIC, Australia

${ }^{2}$ Murdoch Childrens research Institute, Melbourne, VIC, Australia

${ }^{3}$ Victorian Clinical Genetics Services, Melbourne, VIC, Australia

${ }^{4}$ Royal Children's Hospital, Melbourne, VIC, Australia

Spinal muscular atrophy type 1 is a relatively common, untreatable and invariably fatal neuromuscular disorder of early childhood. Family support and genetic counseling form a vital part of the management for families affected by this condition. There is no empirical evidence of how best to support SMA type 1 families during this time. This qualitative study undertook thematic analysis of 11 in-depth interviews with 13 bereaved parents of children with SMA type 1. While individuals' experiences were unique, common themes emerged from the data, including: experiencing shock and anticipatory grief at the time of diagnosis, processing feelings of responsibility and helplessness, experiencing multiple losses including the loss of future reproductive freedom, regaining control by making decisions about the child's life and death, finding peace in the dying process, and feeling well supported. These findings reveal a person/family-centered perspective of the psychosocial impact of having a child with SMA type 1. Health professionals can best support such families by offering grief-specific support beginning from the time of diagnosis, participating in joint decision-making to empower parents, and acknowledging the multiple losses parents may experience.

\section{Oral 17 \\ COMPARING PREFERENCES OF PARENTS, PEDIATRICIANS AND GENETIC HEALTH PROFESSIONALS FOR CHROMOSOMAL MICROARRAY RESULTS}

Erin Turbitt $^{1,2}$, Jane Halliday ${ }^{1,2}$, David Amor ${ }^{1,2}$, Sylvia Metcalfe ${ }^{1,2}$

${ }^{1}$ Murdoch Children's Research Institute, Melbourne, VIC, Australia

${ }^{2}$ The University of Melbourne, Melbourne, VIC, Australia

Background: Genomic chromosomal microarray (CMA) testing for childhood investigations has increased diagnostic yields. However, CMAs also increase detection of incidental findings (IFs) and variants of unknown and uncertain clinical significance (VUS). Elucidating patient disclosure preferences may help clinicians anticipate the type of results about which patients want to be informed. Methods: A questionnaire, using hypothetical scenarios, was designed to investigate and compare the perspectives of parents, pediatricians and genetic health professionals for result disclosure. Quantitative data were analysed using ANOVA and Kruskal Wallis tests. Open text data were analysed using content analysis. Results: 147 parents, 159 pediatricians and 69 genetic health professionals participated and at least $89 \%$ of respondents in each category certainly or probably favored disclosure of VUS as well as variants of certain clinical significance, with the lowest percentage being amongst parents, who were less sure of their disclosure preferences. There was consensus among respondent groups that knowledge of a variant of certain clinical significance would provide more practical and emotional utility compared to VUS. Parents demonstrated some different perspectives to health professionals; for example, they placed more emphasis on using knowledge of a VUS when considering future pregnancies (K.Wallis: $p<.001$ ). Conclusion: This study, together with a previous study investigating the opinions of a subset of these respondents for disclosure of IFs (Turbitt et al., EJHG 2014) is the first Australian exploration of preferences for genomic result disclosure, with implications for clinical practice.

\section{Oral 18 \\ CHILD HEALTH AFTER PREIMPLANTATION GENETIC DIAGNOSIS}

Sharon Lewis ${ }^{1,2}$, Anne Glynn ${ }^{1,2}$, David Amor ${ }^{2,3}$, Leeanda Wilton ${ }^{4}$, Elissa Osborne $^{5}$, Jane Halliday ${ }^{1,2}$

${ }^{1}$ Public Health Genetics, Murdoch Children's Research Institute, Melbourne, VIC, Australia

${ }^{2}$ Department of Paediatrics, University of Melbourne, Melbourne, VIC, Australia

${ }^{3}$ Victorian Clinical Genetic Service, Melbourne, VIC, Australia

${ }^{4}$ Melbourne IVF, Melbourne, VIC, Australia

${ }^{5}$ Monash IVF, Melbourne, VIC, Australia

Despite the increasing use of Preimplantation Genetic Diagnosis (PGD), there is little follow-up of children conceived using PGD beyond 2 years of age. We examined the health, wellbeing and development of school-aged children (aged 5-8 years old) conceived following PGD. A retrospective cohort was undertaken. Children conceived after in-vitro fertilization (IVF) with PGD (exposed cohort) and children conceived after IVF without PGD (unexposed cohort) at two IVF clinics in Melbourne, born between 1999 and 2008 , were recruited with a 1:2 ratio. Mothers of the children completed a questionnaire asking child-specific questions regarding health and wellbeing, mental health, development, educational achievement and family-specific questions regarding family functioning and parent-child attachment. We recruited 155 and 303 participants in the exposed and unexposed cohorts respectively. There were no differences between the cohorts with regards to most child and birth outcomes, birth defects, or maternal variables. However, compared to the unexposed cohort, children in the exposed cohort were more likely to have been delivered by a caesarean section 
$\left(\chi^{2}=11.3, p<.001\right)$, have lower peer problems in the males $(t=$ $2.23, p=.03)$, and lower prosocial behavior in the females $(t=$ $2.9, p<.01)$. While no significant differences between the exposed and unexposed cohorts were found for the majority of psychological scales, there were differences when compared with the normative population data. Children in the exposed cohort appeared to have more positive outcomes in many of the measures. The data suggest that PGD does not causes adverse outcomes in children.

Special Interest Group Day (Sunday): Australasian Society of Genetic Counsellors

\section{FAMILY COMMUNICATION AND NON-DISCLOSURE \\ Carma Bylund' and Clara Gaff \\ ${ }^{1}$ Hamad Medical Corporation, Qatar \\ ${ }^{2}$ Walter and Eliza Hall Institute, Melbourne, VIC, Australia}

Communication within families about genetic conditions is a central issue in genetic counseling. This workshop will use lecture, small group work, and role play to help participants consider how family communication affects their everyday work. In the first part of the workshop, we will explore a theory of family communication patterns and apply it to conversations about genetics. Following, we will address the common problem of active non-disclosure in families and introduce a framework for communicating with families in these situations.

BEING A GENETIC COUNSELOR IN THE UK: TRAINING, REGISTRATION, ROLES

Anna Middleton $^{1,2}$

${ }^{1}$ Wellcome Trust Sanger Institute, Hinxton, Cambridge, UK

${ }^{2}$ Association of Genetic Nurses and Counsellors

This presentation will focus on the infrastructure of genetic counseling in the United Kingdom - how and why we work the way we do. There are approximately 300 genetic counselors (GCs) in the United Kingdom. Our professional body is the Association of Genetic Nurses and Counsellors and it is this group that has created our career pathway and represents the interests of genetic counselors on a national level. In order to practice clinically we have to be registered with the Genetic Counsellor Registration Board. At the moment this is a voluntary registration; as a profession we are in the process of applying for statutory regulation by the British government. Together with the HGSA we have created reciprocal registration pathways so that Australian genetic counselors can easily utilize their training and experience in the British system.

There are four different levels of genetic counselor: trainee GC, GC, principal GC and consultant GC. The majority of genetic counselors work in the National Health Service (i.e. the public sector) in the 23 regional clinical genetics services seeing patients. It is very unusual for genetic counselors to work in private practice as clinical genetics, as a service, only exists in the public sector. However, there are small numbers of genetic counselors attached to private IVF and breast cancer services. Experienced genetic counselors work autonomously with their own case load. I will explore the mechanisms for applying to work in the UK, provide an overview of the sorts of roles available and the salaries that can be expected.
Membership Abstracts/Interesting Cases

\section{EXPERIENCES OF WOMEN WHO CONCEIVED WITH PRE-IMPLANTATION GENETIC DIAGNOSIS WHEN DISCLOSING CONCEPTION TO THEIR CHILD}

Kelly Sullivan', Sharon Lewis ${ }^{4}$, Melody Menezes ${ }^{2}$, David Amor ${ }^{4,3}$

${ }^{1}$ University of Melbourne, Melbourne, VIC, Australia

${ }^{2}$ Monash IVF, Melbourne, VIC, Australia

${ }^{3}$ Melbourne IVF, Melbourne, VIC, Australia

${ }^{4}$ Murdoch Children's Research Institute, Melbourne, VIC, Australia

Background: Preimplantation genetic diagnosis (PGD) is a technique which allows genetic testing on embryos created with in vitro fertilization (IVF). Historically, this has been used for couples with a history of genetic disease, but now a form of PGD known as preimplantation genetic screening (PGS) is often offered to other couples using IVF, as a way to detect any chromosomal imbalances in the embryo. Little is known about if or how parents who use PGD share this information with their children, or if they would benefit from additional support in disclosure. Methods: Previously collected questionnaires from 99 women who conceived with PGD/PGS and IVF and 199 women who conceived with IVF alone were analysed using inductive content analysis. Then, a questionnaire was developed based on those results and 6 women who conceived with PGD/PGS and IVF and had disclosed conception participated in in depth interviews, which were transcribed verbatim and analysed with thematic analysis. Both samples heavily represented the opinions of women who had undergone PGS. Results: Among women who conceived with PGS, disclosure of IVF tends to occur early, but the use of PGS is discussed later in the child's life, or not at all. Women who conceived with PGS questioned the relevance of that information for their child. Women who underwent PGD for familial genetic conditions may have far greater support needs than women who used PGS, as the genetic information has great relevance for the child.

COMPLEX FAMILY RELATIONSHIPS AND CONFIDENTIALITY - AN ETHICAL DILEMMA

\section{$\underline{\text { Sarah Borrie }}$}

SA Clinical Genetics Service, Adelaide, SA, Australia

Hannah was a 27-year-old woman who had a termination of pregnancy for a fetal abnormality. An Xp duplication of uncertain clinical significance was found on autopsy. Several months later, her 29year-old sister, Sandra, was unexpectedly pregnant and contacted the Genetics Service to discuss the risks to her pregnancy. Their father had given Sandra information about Hannah's pregnancy, but Hannah had not given consent to discuss her history. This case explores the clinical geneticist and genetic counselor's strategies to balance Sandra's right to make an informed decision and Hannah's right to privacy and confidentiality. Balancing these rights was also important as the clinical geneticist explored the issue of testing the extended family for the duplication. The difficulties of counselling regarding variants of uncertain significance in the face of decision making were also highlighted.

\section{DECEPTION - FALSE FAMILY HISTORIES AND THE ETHICS} OF CONFIDENTIALITY.

Lindsay Fowles, Rachel Susman and Annette Hattam

Genetic Health Queensland, Royal Brisbane and Women's Hospital, Brisbane, QLD, Australia

When performing a cancer risk assessment, the primary tool is the reported family history. Generally errors in the family history arise 
through family members being mistaken or confused; only rarely do they occur through deliberate falsification. My patient's anxiety regarding her cancer risk was based on her mother's supposed cancer history. As arranged by my patient, her mother reported the family history on her behalf. However, the mother had been seen at the genetics service 16 years previously and irreconcilable discrepancies in the reported family histories were noted. The mother's previous genetics appointment had been to review her cancer risk as she was requesting prophylactic mastectomies. She was now reporting multiple cancer diagnoses in herself, family members previously reported as deceased were now reported as living, and a previously reported daughter with breast cancer was an unrelated step-daughter. Without the mother's written consent to share information, we were left with questions as to what could be disclosed to my patient. The particular characteristics of this case allowed us to avoid a controversial breach of confidentiality; however, it was necessary to explore the ethics of confidentiality to ensure we were properly prepared.

\section{ETHICAL CHALLENGES ASSOCIATED WITH A REQUEST FOR PRENATAL CARRIER TESTING FOR MECP2 DUPLICATION SYNDROME}

\author{
J. Hogan' ', L. Warwick', M. Freckmann ${ }^{2}$ \\ ${ }^{1}$ ACT Genetic Service, Canberra, ACT, Australia \\ 2 Sydney Children's Hospital, Sydney, NSW, Australia
}

Being a female carrier of an X-linked recessive condition usually has no implications for the woman's health. Instead, implications of carrier testing relate to the future reproductive choices for that individual. Carrier testing of children is usually not recommended in the absence of an immediate medical benefit. In addition, prenatal carrier testing presents the challenge of determining the primary patient, as maintaining the future child's autonomy concurrently denies the autonomy of the parent. In our case, a female carrier of MECP2 duplication syndrome requested prenatal carrier testing with the intention of having a termination of pregnancy for an affected male or a carrier female. While males affected by MECP2 duplication syndrome have severe to profound intellectual disability, the majority of female carriers are asymptomatic. The genetic counselor was challenged to deal with the ethical implications of this request and required to develop strategies for resolving the ethical conflict between the counselor and consultand.

UNIQUE CHALLENGES OF THE CARDIAC GENETIC COUNSELOR

Laura Yeates ${ }^{1,2}$, Bronwyn Culling ${ }^{3}$, Raymond Sy², Jodie Ingles ${ }^{1,2,4}$, Christopher Semsarian ${ }^{1,2,4}$

${ }^{I}$ Agnes Ginges Centre for Molecular Cardiology, Centenary Institute, Sydney, NSW, Australia

${ }^{2}$ Department of Cardiology, Royal Prince Alfred Hospital, Sydney, NSW, Australia

${ }^{3}$ HealthShare NSW, Sydney, NSW, Australia

${ }^{4}$ Sydney Medical School, University of Sydney, Sydney, NSW, Australia

$\mathrm{K}$ is a 42-year-old female who presented after recurrent blackouts. Investigations lead to a diagnosis of long QT syndrome (LQTS). LQTS is a primary arrhythmogenic disorder affecting 1 in 3,000 of the population. It is an autosomal dominant disease with 13 genes currently known. Patients with LQTS often present with blackouts and the most serious outcome is sudden cardiac death. Patient $\mathrm{K}$ had clinical characteristics known to predispose to a higher risk of sudden cardiac death, and therefore an implantable cardioverter defibrillator (ICD) was recommended. Within 6 months after implantation, $\mathrm{K}$ disclosed suicide ideation and requested her ICD be turned off. An overview of the case will be covered with a focus on the counseling issues raised and the subsequent interventions required to address these. Discussion will also include the ethical principles of a consultand's autonomous decision conflicting against the ethical principle of non-maleficence. Patient K presents a common scenario in the cardiac genetic setting, whereby coming to terms with the genetic issues is complicated by the issue of sudden cardiac death prevention. There is growing literature focusing on the psychosocial burden of having an ICD and dealing with ICD complications and shocks. The genetic counselor plays a key role in the multidisciplinary care of genetic heart disease patients, particularly those with ICDs. 